\title{
Plant Disease Severity Assessment-How Rater Bias, Assessment Method, and Experimental Design Affect Hypothesis Testing and Resource Use Efficiency
}

\author{
Kuo-Szu Chiang, Clive H. Bock, I-Hsuan Lee, Moussa El Jarroudi, and Philippe Delfosse
}

First and third authors: Division of Biometrics, Department of Agronomy, National Chung Hsing University, Taichung, Taiwan, 402; second author: United States Department of Agriculture-Agricultural Research Service Southeastern Fruit \& Tree Nut Research Laboratory, 21 Dunbar Road, Byron, GA 31008; fourth author: Department of Environmental Sciences and Management, Université de Liège, 185 Avenue de Longwy, 6700 Arlon, Belgium; and fifth author: Luxembourg Institute of Science and Technology, 41 Rue du Brill, L-4422 Belvaux, Luxembourg.

Accepted for publication 13 July 2016.

\begin{abstract}
Chiang, K.-S., Bock, C. H., Lee, I.-H., El Jarroudi, E. and Delfosse, P. 2016. Plant disease severity assessment-How rater bias, assessment method, and experimental design affect hypothesis testing and resource use efficiency. Phytopathology 106:1451-1464.

The effect of rater bias and assessment method on hypothesis testing was studied for representative experimental designs for plant disease assessment using balanced and unbalanced data sets. Data sets with the same number of replicate estimates for each of two treatments are termed "balanced" and those with unequal numbers of replicate estimates are termed "unbalanced". The three assessment methods considered were nearest percent estimates (NPEs), an amended 10\% incremental scale, and the Horsfall-Barratt (H-B) scale. Estimates of severity of Septoria leaf blotch on leaves of winter wheat were used to develop distributions for a simulation model. The experimental designs are presented here in the context of simulation experiments which consider the optimal design for the number of specimens (individual units sampled) and the number of replicate estimates per specimen for a fixed total number of observations

(total sample size for the treatments being compared). The criterion used to gauge each method was the power of the hypothesis test. As expected, at a given fixed number of observations, the balanced experimental designs invariably resulted in a higher power compared with the unbalanced designs at different disease severity means, mean differences, and variances. Based on these results, with unbiased estimates using NPE, the recommended number of replicate estimates taken per specimen is 2 (from a sample of specimens of at least 30 ), because this conserves resources. Furthermore, for biased estimates, an apparent difference in the power of the hypothesis test was observed between assessment methods and between experimental designs. Results indicated that, regardless of experimental design or rater bias, an amended $10 \%$ incremental scale has slightly less power compared with NPEs, and that the H-B scale is more likely than the others to cause a type II error. These results suggest that choice of assessment method, optimizing sample number and number of replicate estimates, and using a balanced experimental design are important criteria to consider to maximize the power of hypothesis tests for comparing treatments using disease severity estimates.
\end{abstract}

Measurement of plant disease severity is central to many applications of plant pathology. For example, measurements of disease severity are needed to compare different methods of disease control (chemical, cultural, host resistance, and so on) and relate to yield loss. The disease severity data on which the relevant analyses are most often based are visual estimates of the relative leaf area showing disease symptoms, and this estimate must be both accurate and reliable (Nutter et al. 1991). However, visual estimates of disease severity as a proportion of area are error prone and affected by several factors (Bock et al. 2010b; Madden et al. 2007; Nutter and Schultz 1995).

Several methods of assessment used to estimate disease severity based on area affected have been explored in previous studies (Bock et al. 2010a; Chiang et al. 2014). The results of the Chiang et al. (2014) study indicated that an amended $10 \%$ category scale with additional grades at low severity can be considered a good choice for assessing disease severity when use of a scale is preferred. Subsequently, the ramifications of rater bias effects on hypothesis testing were also

Corresponding author: C. H. Bock; E-mail address: clive.bock@ars.usda.gov

*The $e$-Xtra logo stands for "electronic extra" and indicates that eight supplementary figures are published online.

http://dx.doi.org/10.1094/PHYTO-12-15-0315-R

This article is in the public domain and not copyrightable. It may be freely reprinted with customary crediting of the source. The American Phytopathological Society, 2016. studied (Chiang et al. 2016). The results of the latter study demonstrated that the power of the hypothesis test is greatest when estimates are unbiased. The power of the hypothesis test depends on the type II error rate (power $=1-$ type $I$ I error rate). A type II error is defined as the probability of accepting the null hypothesis $\left[\mathrm{H}_{0}\right]$ when $\mathrm{H}_{0}$ is false, and a type I error is the probability of rejecting $\mathrm{H}_{0}$ when $\mathrm{H}_{0}$ is true. Thus, rater bias increases the type II error rate; the effect of rater bias was even greater than the increase in type II error due to use of certain assessment methods, including some category scales (Chiang et al. 2016). Thus, it is important to investigate whether any approaches can reduce errors, particularly when disease severity is in the 1 to $10 \%$ range, a range known to be particularly prone to overestimation (Bock et al. 2010b; Godoy et al. 2006; Sherwood et al. 1983). For example, in relation to experiments with comparisons of disease severity, it is important to understand whether there are experimental designs that reduce the power of the hypothesis test compared with others, and to understand how these might interact with biases in estimation of severity or with the method selected to measure severity.

As noted above, error of estimates can be particularly profound in the 1 to $10 \%$ severity range. This is a particularly important range for many epidemiological studies because it is disease at low severity that forms the basis for obtaining parameters that might be used in projecting epidemic development, consequent yield loss, and the need to treat with pesticides. It is also a severity range over which treatments might be compared (for example, fungicide efficacy and host resistance). It is particularly relevant to consider 
the interactive effects of bias, assessment methods, and different experimental designs on the outcome of the analysis for which these severity data are used.

A disease assessment scheme should, ideally, be reliable and accurate (Campbell and Madden 1990; Madden et al. 2007; Nutter and Schultz 1995). Reliability can be defined as "the extent to which the same measurement of individuals obtained under different conditions yield similar results" (Everitt 1998). In studies of plant disease assessment, there are two aspects of reliability that need to be considered. The first is intrarater reliability, or how similar repeat measurements taken by the same rater are to one another, which is also known as repeatability (Nutter et al. 1991). The second is interrater reliability, which is how similar measurements of the same specimens are between or among raters or rating methods at the same time (Bock et al. 2010b). Our study focused on experimental designs in relation to intrarater reliability.

In various studies of plant disease assessment, there are examples in which replicate estimates of severity of sets of individual leaves by individual raters have been used to evaluate intra- and interrater reliability (Bock et al. 2008a,b; Duarte et al. 2013; Nita et al. 2003; Nutter et al. 1993; Shokes et al. 1987). In assessing experiments, any individual rater should assess complete replicates of all treatments so as to spread any rater-inherent bias or imprecision evenly across those treatments (Bock et al. 2015). In general, the purposes of replicates are to provide an estimate of experimental error, improve the precision of the mean estimates of an experiment, increase the scope of inference of the experiment, and control for error variance (Steel et al. 1997).

In statistics, data sets with the same number of replicate estimates for each of two treatments are termed "balanced" and those with unequal numbers of replicate estimates are termed "unbalanced". To the best of our knowledge, a comparison of balanced and unbalanced data sets in experimental designs has not previously been tested in relation to disease assessment methods and rater bias.

Due to resource limitations, scientists are often interested in developing the optimal experimental design in which the number of specimens (individual units sampled) and the number of replicate estimates per specimen for a fixed total number of observations (total sample size for the treatments being compared) are chosen to maximize statistical power and efficiency. Thus, apart from ability to estimate disease accurately and reliably, the efficiency of resource (labor, time, and money) use should be maximized. In medical science, both sampling and experiment design have been considered to ensure best resource use while maximizing the precision of the estimate of the intraclass correlation coefficient (ICC). The reliability of continuous outcome measures is usually assessed by estimation of the ICC (Giraudeau and Mary 2001; Shoukri 2004; Shoukri et al. 2003). In these studies, it has been ascertained that a higher number of replicate estimates would lead to a much smaller number of specimens and, hence, loss in precision of the estimated reliability; in sampling, two to three measurements per specimen are recommended (rather than taking additional specimens, which is resource demanding and, thus, costs more). In addition to considering the aforementioned efficiency requirements, some medical studies further investigated how funding constraints determine the recruiting cost of specimens needed for a reliability study (Eliasziw and Donner 1987; Flynn et al. 2002; Shoukri 2004; Shoukri et al. 2003). That is, the objective of the studies is to collect reliable data for testing purposes (thus, the need is to consider reliability and cost, selecting the number of specimens and replicate estimates per sample to ensure sufficient reliability or agreement while minimizing cost). The results of the above studies involving cost implications demonstrated that the optimal allocation for replicate estimates per sample is only two or three replications per subject on most occasions. In plant pathology, the goal is most often to compare treatments. However, experimental design and number of specimens versus number of replicates per specimen have rarely been considered in plant disease assessment. Based on the results we present, there is no reason that the same concept applied in medical research should not be used in experiments involving plant disease severity estimation.

The current disease assessment paradigm in plant pathology is to take only a single estimate for each specimen. The effects of experimental design or the possibility of taking multiple estimates of each specimen have not been considered previously in published studies of visual assessment methods for plant diseases. Only effects of rater bias and assessment method have been explored (Bock et al. 2010a; Chiang et al. 2014, 2016). In this study, we use simulation to explore the effects of experimental design by replicating estimates of individual specimens (in relation to sample size). We also consider how these effects are interrelated with rater bias and assessment method, and quantify the effect on hypothesis testing. Furthermore, this study explores the optimal design for the number of specimens (individual units sampled) and the number of replicate estimates per specimen for a fixed total number of observations (total sample size for the treatments being compared). The results suggest that choice of assessment method, optimization of sample number, number of replicate estimates, and whether or not the experiment has a balanced design are all important criteria to consider when attempting to maximize the power of hypothesis tests for comparing treatments using disease severity estimates.

Thus, the specific objectives of these simulations were to (i) compare balanced and unbalanced experimental designs when basing analysis on disease severity data; (ii) determine the effect of the number of replicate estimates per specimen (i.e., multiple estimates of the same specimen) for a fixed total number of observations; and (iii) address how sample size, severity estimation bias, and assessment method interact with experiment design and replicate number, and how this affects the probability of a type II or type I error.

\section{MATERIALS AND METHODS}

Data used to develop distributions for the simulation model. We describe a series of simulations of plant disease severity to investigate the influence of experimental design. The simulations are based on data previously described (Chiang et al. 2016; El Jarroudi et al. 2015). For this data set, the rater-estimated and actual severity data were from samples of leaves of winter wheat with symptoms of Septoria leaf blotch (SLB, caused by Zymoseptoria tritici (Desm.) Quaedvl. \& Crous) that were collected from plants in both plots receiving fungicides and control plots (no fungicide spray) in field experiments in the Grand-Duchy of Luxembourg. For estimates of disease severity, visual nearest percent estimates (NPEs) of SLB and SLB-associated senescence were made on the flag leaf (F1), and on the two leaves below the flag leaf (F2 and F3) by four raters. A total of 20 plants for each treatment was assessed on each assessment date (El Jarroudi et al. 2015). Images of the collected leaves used for the visual assessments were taken with a digital camera, and the area with symptoms of SLB and associated senescence was measured by image analysis (Assess V2.0). These measurements were the assumed actual values for disease severity on each leaf (it is acknowledged that even measurements by image analysis are subject to some error but they are considered more accurate than other methods) (Bock et al. 2008a; Martin and Rybicki 1998). In total, 345 leaves in 2006 and 201 leaves in 2007 from nontreated plots were photographed, image analyzed, and assessed; 240 leaves in 2006 and 171 leaves in 2007 from fungicide-treated plots were subjected to the same procedure (a grand total of 957 leaves).

Assessment methods. The following assessment methods were compared:

i. NPEs (disease severity estimated by the raters to the nearest $1 \%)$

ii. An amended $10 \%$ incremental scale with additional grades at low severities $(0.1,0.5,1.0,2.0,5.0,10.0,20.0,30.0 \ldots 100 \%)$ (Chiang et al. 2014);

iii. The Horsfall-Barratt (H-B) scale (Horsfall and Barratt 1945). 
The NPEs were converted to the appropriate grades for assessment methods 2 and 3 for the simulations (Bock et al. 2010a; Chiang et al. 2014; Nita et al. 2003). The scale data were subsequently converted to the appropriate category midpoint value of each grade for analysis (Madden et al. 2007).

Bias and simulations. Actual values (measured by image analysis) and estimates by the four different raters of the severity of SLB were used to develop distributions describing unbiased and biased effects for a simulation model.

A linear model was used in order to describe the relationship between the mean rater estimated severity $\left(\mu_{\text {rater }}\right)$ and the actual severity ( $Y_{\text {actual }}$ ) (Bock et al. 2010a; Chiang et al. 2014). Moreover, the standard deviation of the rater mean NPE $\left(\sigma_{\text {rater }}\right)$ was regarded as a function of $Y_{\text {actual }}$ determined by the rater estimates of severity of SLB on leaves of winter wheat. The relationships are as follows:

$$
\mu_{\text {rater }}=\theta Y_{\text {actual }}
$$

and

$$
\sigma_{\text {rater }}=f\left(Y_{\text {actual }}\right)
$$

In previous articles (Bock et al. 2010a; Chiang et al. 2014; Forbes and Korva 1994), the frequency of NPEs of specific actual disease severities by raters was assumed to follow a lognormal distribution. That is:

$$
y_{\mathrm{i}} \sim \operatorname{Lognormal}\left(\mu, \rho^{2}\right)
$$

The lognormal distribution is a positively skewed distribution. Usually, disease estimates at low actual severities have a positively skewed distribution (i.e., overestimation was considered). Conversely, a negatively skewed distribution (i.e., 1 - lognormal distribution) is usually observed for estimates at high disease severities (i.e., underestimation was considered) (Fig. 1). The SLB data set used in this study confirms this characteristic. The two parameters of equation 3 were derived from equations 1 and 2 . Their relationships are as follows (Bock et al. 2010a; Chiang et al. 2014):

$$
\mu=\ln \left(\mu_{\text {rater }}\right)-\frac{1}{2} \ln \left[1+\left(\frac{\sigma_{\text {rater }}}{\mu_{\text {rater }}}\right)^{2}\right]
$$

and

$$
\rho=\sqrt{\ln \left[1+\left(\frac{\sigma_{\text {rater }}}{\mu_{\text {rater }}}\right)^{2}\right]}
$$

For the unbiased situation, $\theta$ in equation 1 is constant and equals 1 . However, for the biased situation, $\theta$ is not constant because the effects of rater bias are different for individual raters. Also, if the same rater assesses the same leaf several times, the results are expected to be variable. Here, we used the simulation model to sample a large data set of rater-estimated disease severities. First, $\theta$ is assumed to be 1 and, to account for this uncertainty in degree of bias, a large number of simulation values were produced for $\theta$. Afterward, if the effect of rater bias was overestimation (or underestimation), we drew only random samples with values greater (or less) than the mean of a lognormal distribution (or a 1 - lognormal distribution) in order to represent the effect of the overestimation (or underestimation) (Fig. 1). By using the simulated data, effects of biased estimates can be explored.

In order to quantify the relationship between the standard deviation of the mean estimated severity and the actual severity in equation 2, unbiased and biased situations were considered (Fig. 2). The four raters who assessed SLB on wheat leaves represent different hypothetical rater types used in the study. The characteristics of the rating abilities of these four raters have been described previously (Bock et al. 2015; El Jarroudi et al. 2015); they covered a spectrum of ability to overestimate except at extremely high severities (raters 3 and 4), underestimate (rater 2), and show relative accuracy of estimation (rater 1), thereby providing a fair representation of the rater population. With the example of an unbiased rater, the relationship was obtained using the estimates of rater 1 from the SLB data set (Fig. $2 \mathrm{~A})$. For the situation where raters overestimated severity for low and midrange actual severities, the relationship was based on the estimates of raters 3 and 4 from the same data set (Fig. 2B) (Bock et al. 2015; El Jarroudi et al. 2015). Because raters 2, 3, and 4 tended to underestimate SLB severity at high actual severities, the data from raters 2,3 , and 4 were used to depict the relationship (Fig. 2C). To consider the ramifications of underestimation at midrange actual severity (50\%), only the data from rater 2 was adopted because only rater 2 tended to underestimate SLB severity in this actual range (Fig. 2D). For each of the scenarios shown (Fig. 2), the data were subject to polynomial curve fitting, and a parabolic curve was found to best describe the relationship between the standard deviation of the rater mean NPE $\left(\sigma_{\text {rater }}\right)$ and the actual disease severity. The parameters, the corresponding standard error, and the coefficient of determination $\left(R^{2}\right)$ for each of the scenarios were used to evaluate the appropriateness of the parabolic model. The relationship between the standard deviation of the rater mean NPE and the actual disease severity for unbiased and biased estimates has a parabolic relationship $\left(\sigma_{\text {rater }}=a Y^{2}\right.$ actual $\left.+b Y_{\text {actual }}+c\right)$ (Fig. 2A to D), reflecting the characteristics of disease severity estimation of the raters of differing ability. The analyses were performed using SAS (SAS V9.3; SAS Institute, Cary, NC).

The steps in the simulation process were as follows:

i. First, an actual severity $\left(Y_{\text {actual }}\right)$ value for a treatment was selected.

ii. Second, the rater-estimated severity $\left(\mu_{\text {rater }}\right)$ and standard deviation $\left(\sigma_{\text {rater }}\right)$ were calculated using equations 1 and 2 .

iii. Third, $\mu_{\text {rater }}$ and $\sigma_{\text {rater }}$ values were substituted into equations 4 and 5 to acquire the parameters $\left(\mu\right.$ and $\left.\rho^{2}\right)$ of the lognormal

A

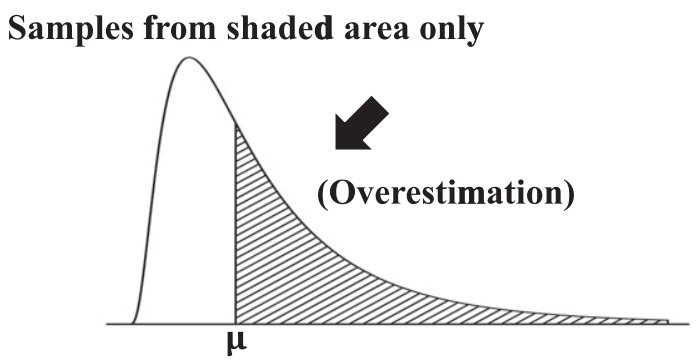

Low severities

B Samples from shaded area only

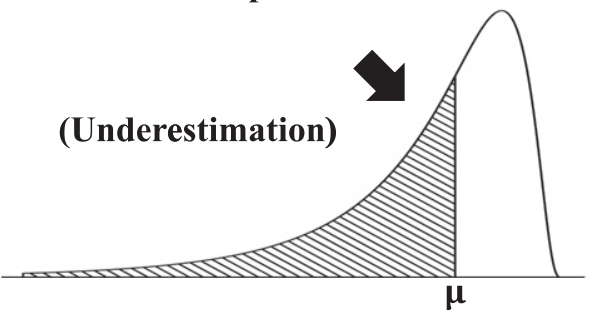

High severities

Fig. 1. A, Lognormal distribution is a positively skewed distribution. The lognormal distribution of disease severity used for the simulation study at low actual severities where raters were either unbiased (all of the area under the curve) or overestimated (the shaded area). B, There is a $1-$ lognormal distribution (a negatively skewed distribution) at high disease severities. The $1-\operatorname{lognormal}$ distribution of disease severity used for the simulation study at high actual severities where raters were either unbiased (all of the area under the curve) or underestimated (the shaded area). Samples were taken from the corresponding areas. Mean severity $=\mu$. 
distribution in equation 3, representing severity as assessed by a generalized rater base on the SLB data.

iv. Finally, a simulated value based on the distribution of raterestimated disease severities was obtained using equation 3 .

Simulated data for different experimental designs. How rater bias and assessment method used to estimate disease severity affect hypothesis testing in different experimental designs was investigated. Through simulation experiments, we considered the different distributions between the number of specimens and the number of replicate estimates per specimen for a fixed total sample size for both treatments being compared. Thus, several combinations were compared. For example, if a fixed total number of observations is assumed to be 120 for both treatments $(A+B)$, then there are six different combinations of $\mathrm{A}$ and $\mathrm{B}$ that might be balanced or unbalanced: that is, $(1,1),(2,2),(3,1),(4,1),(3,3)$, and $(5,1)$. The corresponding numbers of leaves for treatment $\mathrm{A}$ and $\mathrm{B}$ are $(60,60),(30,30),(90,30),(96,24),(20,20)$, and $(100,20)$, respectively. These contrasting approaches will involve sampling at different intensities and, thus, affect how much resource is required. The fewer specimens sampled, the less resource required.

To account for different combinations between the number of specimens and the number of replicate estimates per specimen for a fixed total number of observations (total sample size for both treatments being compared), the number of specimens sampled ranged from 20 to 180 , which is a range of specimens commonly encountered in experiments in plant pathology. For example, Todd and Kommedahl (1994) used a randomized block design with four replicate estimates of 10 plants per treatment, which was repeated three times. In addition, Parker et al. (1995) compared three seed treatments to control powdery mildew on winter barley and used a group of nine raters to estimate the severity of $S$. tritici and leaf senescence on 25 to 34 naturally infected leaves collected from the field. Bock et al. (2015) described a total sample size of 60 leaves consisting of 3 leaves/plant on each of 5 plants/plot.

Specimens sampled in our simulation studies were assumed to number at least 20 . The rationale is based on the assumption that a certain minimum number of specimens sampled allows the central limit theorem to apply when the $t$ test is used to test the null hypothesis. Although a nonparametric approach is a feasible method, its efficiency is less than that of the $t$ test (Bock et al. 2010a). The central limit theorem states that, for large samples, the sampling distribution of the mean can be approximated to a normal distribution. The question remains: how large a number of specimens must be collected so that the central limit theorem can be applied? This is a fundamental issue which investigators often have to address. From a statistical point of view, sample sizes of 30 or more are generally regarded as sufficiently large, unless the population has a very unusual distribution (Freund and Perles 2006).

Hypothesis testing. The performance of different assessment methods was compared. Assuming that two treatments (treatments $\mathrm{A}$ and $\mathrm{B}$ ) are applied to developing epidemics, the disease severity distribution of treatment $\mathrm{A}$ has mean $\mu_{\mathrm{A}}$ and treatment $\mathrm{B}$ has mean $\mu_{\mathrm{B}}=\mu_{\mathrm{A}}+\mu_{\Delta}$, where $\mu_{\Delta}$ represents the difference between the means of the two severity distributions. The criterion used to gauge the optimal combinations is the power of the hypothesis test for comparing treatments. Hypothesis testing requires that the collected data be sufficiently accurate to reject the null hypothesis $\left(\mathrm{H}_{0}\right)$ when $\mathrm{H}_{0}$ is false or, conversely, to accept $\mathrm{H}_{0}$ when there are no treatment differences. Failure to reject $\mathrm{H}_{0}$ when $\mathrm{H}_{0}$ is false results in commission of a type II error $(P[\mathrm{TII}])$, while rejection of $\mathrm{H}_{0}$ when $\mathrm{H}_{0}$

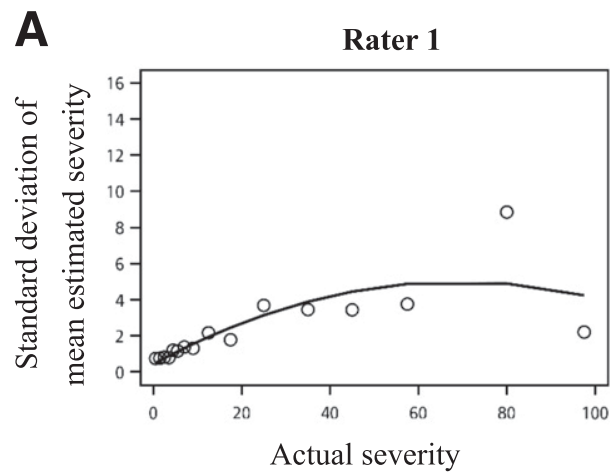

B

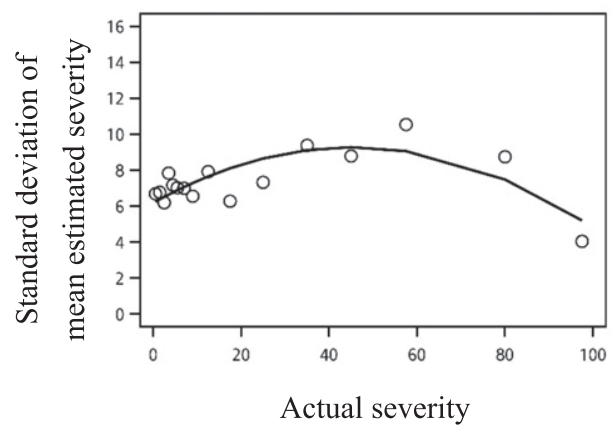

C

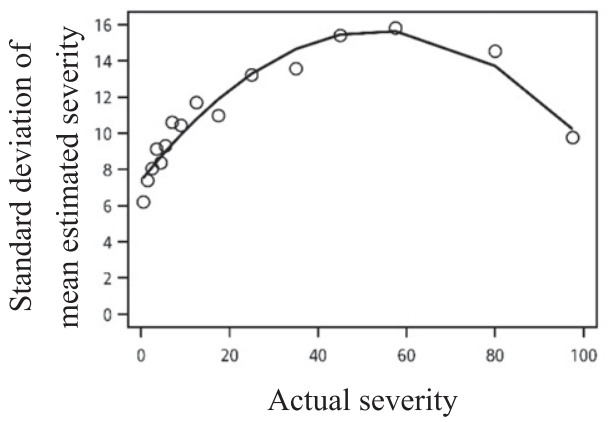

Raters 2

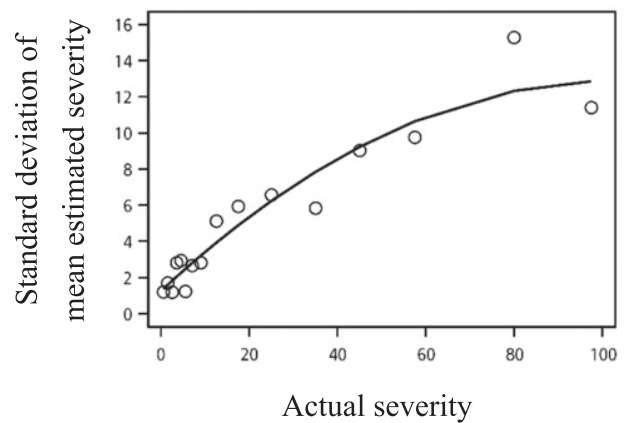

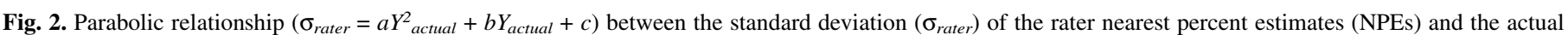

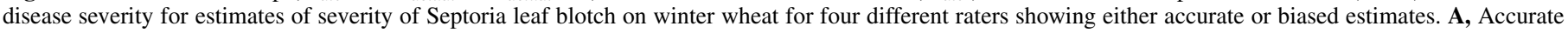

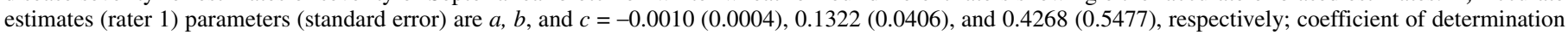

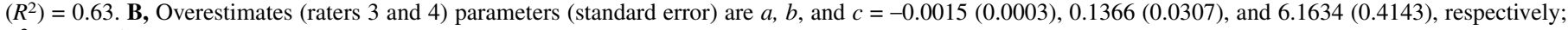

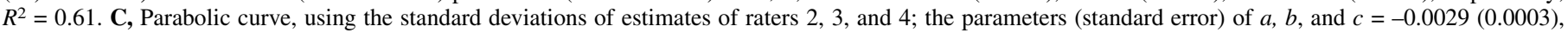

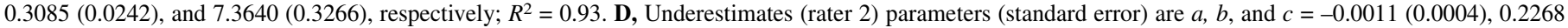
(0.0392), and $1.2611(0.5286)$, respectively; $R^{2}=0.92$. 
is true results in commission of a type I error $(P[T I])$. The power of the hypothesis test is a complement of a type II error. Type II and type I errors might arise from inaccurate rater estimates of severity. To calculate the probability that $\mathrm{H}_{0}$ is rejected, the simulation procedure outlined above was repeated 10,000 times and a $t$ test performed on samples from treatment $\mathrm{A}$ and treatment $\mathrm{B}$ with each simulated data set. For low disease severities, two severities $\left(\mu_{A}\right)$ of 5 or $20 \%$ were used to explore effects on type II and type I error rates. The difference between the population means $\left(\mu_{\Delta}\right)$ was assumed to be 5 or $10 \%$. For high disease severities, actual severities $\left(\mu_{\mathrm{A}}\right)$ of 75 or $90 \%$ were selected. To represent a midrange actual severity, a $\mu_{\mathrm{A}}$ of $50 \%$ was considered. The population disease severity standard deviation $(\varphi)$ was equal to 5 or $10 \%$. The total sample sizes $(N)$ for both treatments being compared are 120, 240, and 360. The significance was $P=0.05$. Simulations were computed and the results of the hypothesis test plotted in relation to the various parameters.

\section{RESULTS}

Low disease severities. Effects of different experimental designs. As expected, for a disease severity mean of $20 \%$ at a given fixed total number of observations, the balanced experimental design resulted in higher power compared with the unbalanced design (Fig. 3). For example, the powers of $(2,2)$ versus $(3,1)$ and $(3,3)$ versus $(5,1)$ were invariably greater for the overestimated data at a sample size $(N)$ of 120 (Fig. 3D). Thus, in a balanced experimental design where the number of specimens collected equals 30 and two replicate estimates are taken per specimen, the total sample size for the two treatments being compared equals 120 .

With a balanced experimental design, more replicates per specimen are taken at the cost of fewer specimens. The greater the number of replicate estimates per specimen, the lower the power of the hypothesis test (Fig. 3A). This effect was even more apparent with overestimates (Fig. 3D). Although the highest power occurs with one replicate per specimen, the design involves more cost to collect a greater number of individual specimens. Moreover, when the total sample size $(N)$ for the sum of both treatments increases, the difference of the power between $(1,1)$ and $(2,2)$ is not discernable. For the unbiased situations shown in Figure 3, there is a similar power with experimental designs of $(1,1)$ and $(2,2)$ with total sample sizes up to approximately 120 for NPEs. Thus, using the power of the hypothesis test as a criterion, two replicate estimates taken per specimen will increase the efficiency of resource use while maintaining the same statistical power for NPEs (Fig. 3A to C).

The effects of a total sample size of 240 (Fig. 3B, which shows the situation with unbiased estimates) demonstrates that the power of the experimental designs $(1,1),(2,2),(3,3)$, and $(4,4)$ is similar for NPEs. In this situation, $(4,4)$ indicates that the number of specimens collected equals 30 and four replicate estimates are taken per specimen. However, the number of specimens collected might be too small to represent the disease severity in that field, especially if the field is large. Thus, taking two replicate estimates per specimen will be safe.

In a real situation, and considering overestimation when using the $10 \%$ incremental scale, to obtain a higher power, the total sample size for the treatments being compared should be increased to approximately 240 (Fig. 3E). When the number of specimens collected equals 60 and two replicate estimates are taken per specimen, the power approaches $100 \%$.

For unbalanced data, the greater the difference in replicate numbers between the treatments, the lower the power of the hypothesis test. For example comparing $(3,1)$ versus $(4,1)$ or $(5,1)$ shows the effect on the power of the hypothesis test, which is especially pronounced with overestimates (Fig. 3D, E, and F). Not unexpectedly, the power of the hypothesis test increases with total sample size $(N=120$ versus 240 versus 360 for either biased or unbiased estimates) (Fig. 3).

The ramifications of smaller sample sizes were also explored (Fig. 4). The difference in the power of experiment designs $(1,1)$ and $(2,2)$ is more apparent due to effects of the total sample size as it is reduced below $N=120$. For example, a comparison of the effects of a total sample size $N=60$ (Fig. 4A, showing the situation with unbiased estimates) with the effects of a total sample size of $N=120$ (Fig. 4D) demonstrates that the power of the experimental designs $(1,1)$ and $(2,2)$ is reduced to 0.9 and 0.7 , respectively when $N=60$ for NPEs, whereas the powers of the experimental designs $(1,1)$ and $(2,2)$ are both $>0.95$ when $N=120$ for NPEs. Also, when the total sample size for the treatments being compared increases from 60 to 120, the power will increase (Fig. 4A to D). To ensure adequate power when two replicate estimates are taken per specimen, the total sample size must be at least 120 (i.e., 30 specimens, two replicate estimates, and two treatments) for NPEs. The result supports our recommendation that the number of specimens collected should be larger than 30 (when taking two estimates per specimen).

Effect of overestimates and assessment methods as dependent on experiment design. The effects of overestimates on the power of the hypothesis test are the same as those for the unbiased estimates, although the overestimates have slightly lower power. The differences in power between assessment methods is more pronounced for overestimates as compared with the unbiased estimates (Fig. 3D versus A or E versus B). Regardless of experimental design, using the $\mathrm{H}-\mathrm{B}$ scale is consistently most likely to cause a type II error, whereas the power of the amended $10 \%$ scale has greater congruence with that of NPEs (although it has slightly less power compared with NPEs).

Effect of population and assessment parameters on the power of the tests as dependent on experiment design. As might be expected, a larger population $\varphi$ results in a decline in the power of the hypothesis test (compare data in Figure 5 , where the $\varphi$ is $10 \%$, with data in Figure 3 , where $\varphi=5 \%$, with all other parameters the same). If population variability is large, differences in the effects of the assessment methods will be relatively small compared with sample variability, regardless of the experimental designs or rater biases. Furthermore, differences among assessment methods and effects of rater bias are less evident at larger population $\varphi$. With a shift in mean population difference $\left(\mu_{\Delta}\right)$ from 5 to $10 \%$, the power of hypothesis tests becomes greater (very near 1.0, regardless of experimental design or sample size) (Fig. 6). Only the $\mathrm{H}-\mathrm{B}$ scale at $N=120$ and replicate estimates of $(4,1),(3,3)$, and $(5,1)$ showed a slight reduction in power compared with the other methods. Thus, as $\mu_{\Delta}$ is increased $>10 \%$, the power of the test approaches 1.0 for all experimental designs, methods, and sample sizes tested. In the case where both the $\varphi$ and $\mu_{\Delta}$ are assumed to be $10 \%$ and the disease severity mean is $20 \%$ (Fig. 7 ), the power of the hypothesis tests falls between those indicated in Figures 5 and 6 . Thus, the result represents the effects of both increasing the population $\varphi$ and changing the $\mu_{\Delta}$, as would be expected.

In general, the trends in the power of the hypothesis tests are very similar at severity $\left(\mu_{\mathrm{A}}\right)$ of $5 \%$ (Supplementary Figs. S1 to S4) to those at $\mu_{\mathrm{A}}$ of $20 \%$ for different experimental designs (already described above; Figures 3, 5, 6, and 7, respectively). Neither sample size nor experimental design appeared to have an effect at $\mu_{\mathrm{A}}=5$ or $20 \%$, regardless of population $\varphi$ or $\mu_{\Delta}$ tested. However, at $\mu_{\mathrm{A}}=$ $5 \%$, the differences between assessment methods are scarcely apparent. Thus, there is similar power for the H-B scale compared with the other assessment methods (for example, Supplementary Figure $\mathrm{S} 1$ versus Figure 3 ). With the exception of the severity mean of $5 \%$, all other parameters used for the simulations in Supplementary Figures S1 to S4 are the same as for the severity mean of $20 \%$ illustrated in Figures 3, 5, 6, and 7, respectively.

Effect on type I errors. When there is no difference between the two population severity means, and the analysis of the sample data results in a significant difference, a type I error is committed. Differences in experimental designs and in assessment methods had virtually no effect on type I errors for these data whether unbiased or overestimated (Supplementary Figs. S5 to S8).

High disease severities. For a disease severity mean of $75 \%$, the trends are similar to those at $20 \%$ (Fig. $8, \mu_{\Delta}$ of $5 \%$ and population $\varphi$ of $5 \%$ ). However, the power of the hypothesis test declined regardless of experimental design, assessment method, or rater bias. 
The decline is due to the relationships between the $\varphi$ of the rater mean NPE and the actual disease severity at a severity of $75 \%$ compared with that observed at $20 \%$. There are larger $\varphi$ of rater means at high disease severities compared with low disease severities. At a severity of $90 \%$ (data not shown), the relationships were similar to those at $5 \%$ severity. Furthermore, the different experimental designs, rater bias, and assessment methods had virtually no effect on type I errors at the high disease severity tested (data not shown).
A

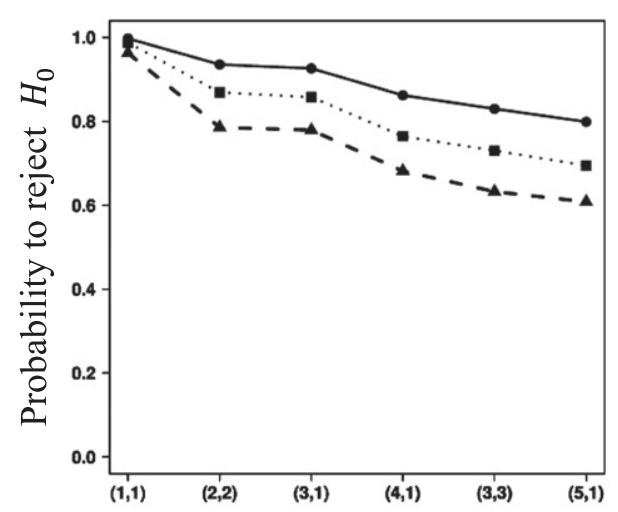

B

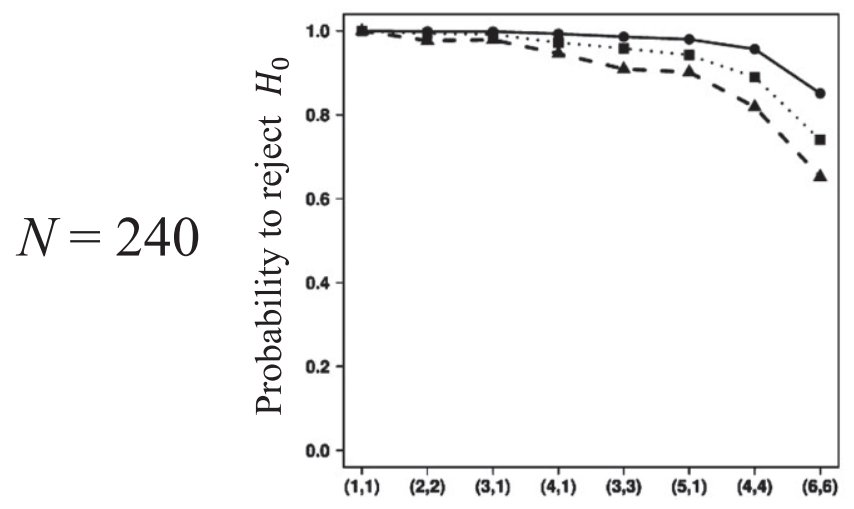

C
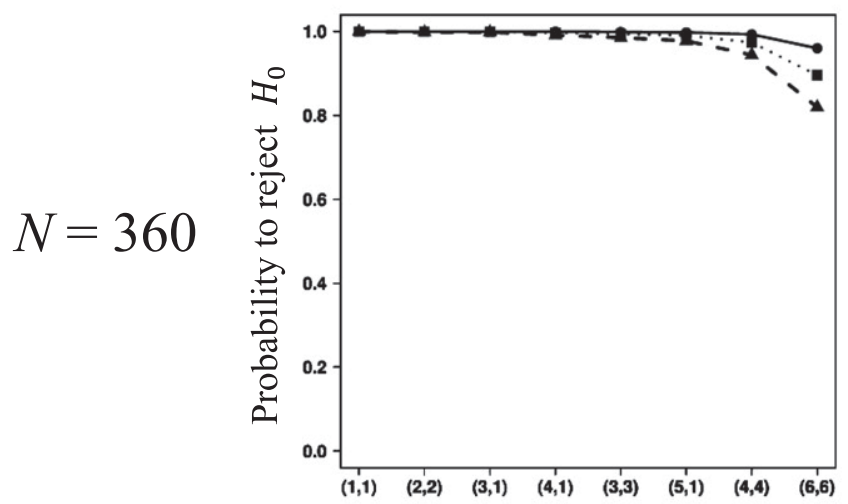

Experimental design
D Overestimated

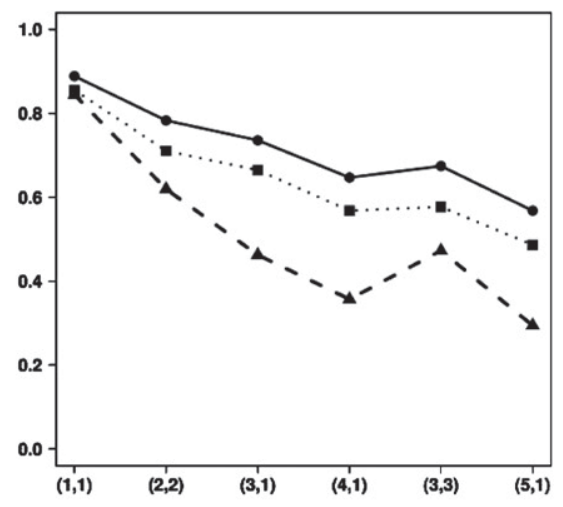

$\mathbf{E}$

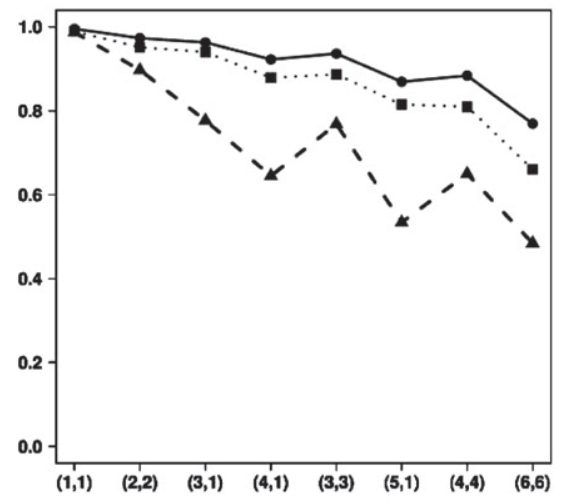

$\mathbf{F}$

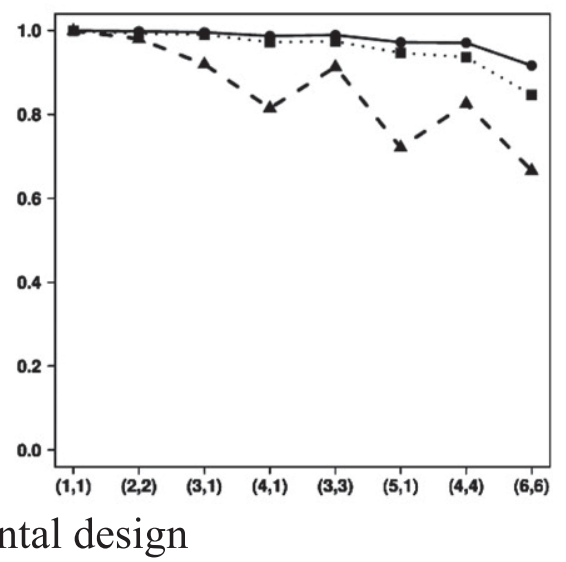

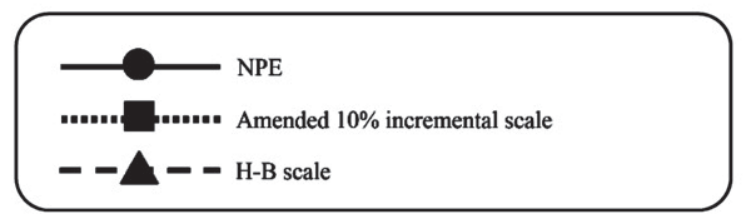

Fig. 3. Relationships between the probability of rejecting the null hypothesis $\left(\mathrm{H}_{0}\right.$, when this hypothesis is false) and different experimental designs (in this study, using $x, y$ indicates that the number of replicate estimates for treatments A and $\mathrm{B}$ are $x$ and $y$, respectively) for the three different assessment scales under different scenarios: A, total sample size of 120 and unbiased; B, total sample size of 240 and unbiased; C, total sample size of 360 and unbiased; D, total sample size of 120 and overestimated; $\mathbf{E}$, total sample size of 240 and overestimated; and $\mathbf{F}$, total sample size of 360 and overestimated. NPE $=$ nearest percent estimates and H-B = Horsfall-Barratt. Difference between the population means $\left(\mu_{\Delta}\right)$ is assumed to be $5 \%, \varphi=5 \%$, and $\mu_{\mathrm{A}}=20 \%$, with significance at $P=0.05$. 
Midrange disease severities. The effects of the different experimental designs, rater bias, and assessment methods on the power of the hypothesis test at the midrange (tested at $\mu_{\mathrm{A}}=50 \%$ severity) were generally similar to the effects at low disease severities (Fig. 9, $\mu_{\Delta}$ of $5 \%$ and population $\varphi$ of $5 \%$ ). However, the power of balanced versus unbalanced designs- $-(3,3)$ versus $(5,1)$ and $(2,2)$ versus $(3,1)$ - was not always greater with the overestimated and underestimated data when using the H-B scale (Figs. 9D and 9G). Moreover, when different $\mu_{\mathrm{A}}, \mu_{\Delta}$, and $\varphi$ were used, the trends of the results were similar to those shown in Figure 9. There was virtually no effect on type I error rates (data not shown).

\section{DISCUSSION}

The results of these simulation studies demonstrate that experimental designs, rater bias, and assessment methods all affect type II error rates. However, these factors had virtually no effect on type I error rates.
Experimental design in relation to hypothesis testing. Using a balanced experimental design dramatically increases the power of the hypothesis test. This observation is consistent with established statistical knowledge and has been summed up in a statement by Montgomery (2005) that "the power of the test is maximized if the samples are of equal size". In any experiment, it is important to have the correct degree of precision and, according to our results, it is preferable to use balanced data and to ensure selection of an optimal number of replicate estimates and a sample size sensitive to the expected means, mean difference, and variance.

A novel and valuable insight from these results is the observation that, in the situation with unbiased estimates using NPEs, taking two estimates per specimen (from a sample of at least 30 specimens) will increase the efficiency of resource use while maintaining the same statistical power as that obtained for a total sample size of 120 specimens. The power for three or four replicate estimates taken per specimen is also high for a total sample size of over 240 specimens. However, in some situations, the number of specimens collected might

\section{Unbiased}
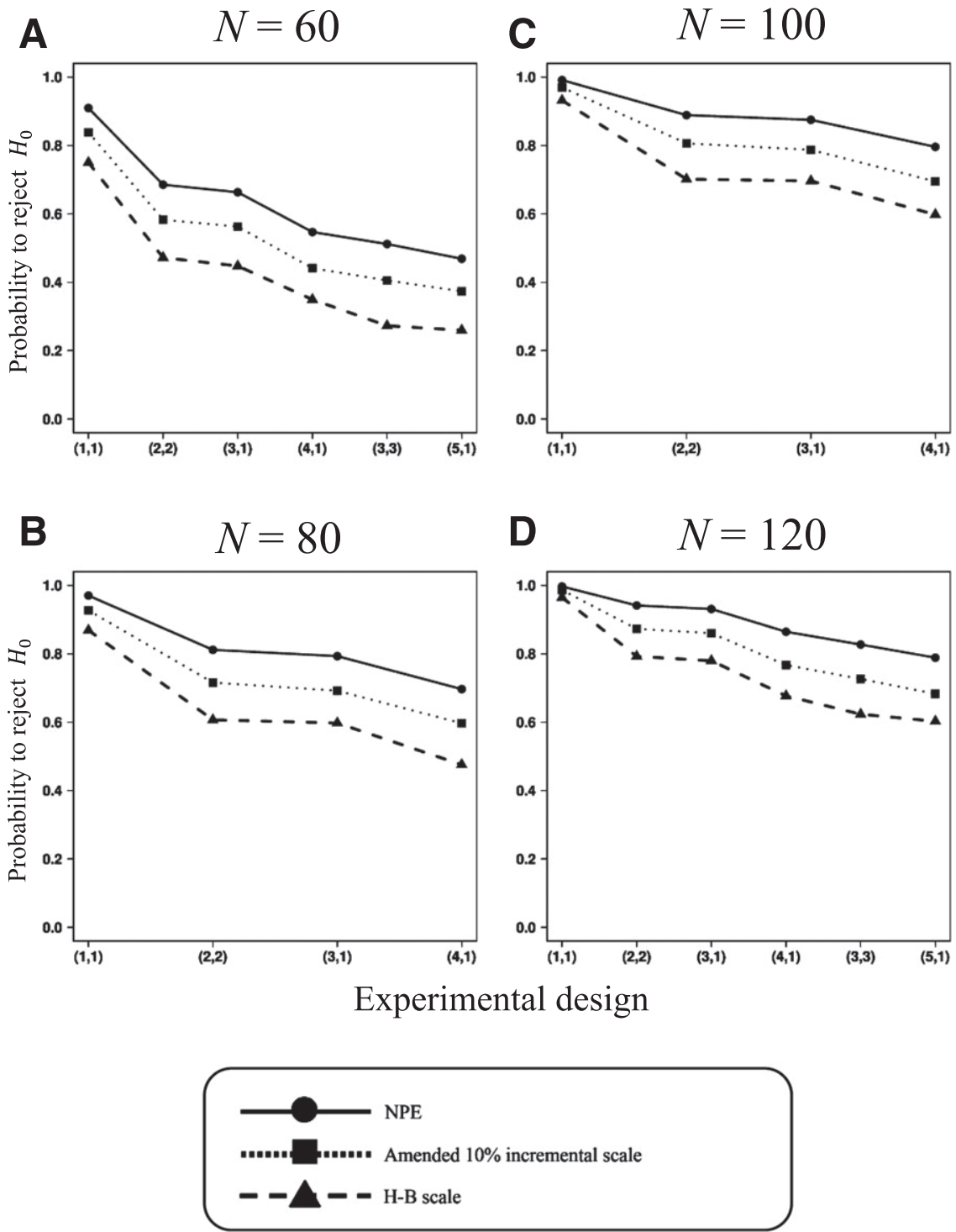

Fig. 4. Relationships between the probability of rejecting the null hypothesis $\left(\mathrm{H}_{0}\right.$, when this hypothesis is false) and different experimental designs (in this study, using $x, y$ indicates that the number of replicate estimates for treatments A and B are $x$ and $y$, respectively) for the three different assessment scales and unbiased estimate under four scenarios: A, B, C, and $\mathbf{D}$, sample sizes of 60,80,100, and 120, respectively. (D is the same as Fig. 3A). NPE $=$ nearest percent estimates and $\mathrm{H}-\mathrm{B}=$ Horsfall-Barratt. Difference between the population means $\left(\mu_{\Delta}\right)$ is assumed to be $5 \%, \varphi=5 \%$, and $\mu_{\mathrm{A}}=20 \%$, with significance at $P=0.05$. 
be too small to represent the disease severity in the population accurately (for example, in a large field). Thus, the recommendation of two replicate estimates taken per specimen will be safe in most sampling situations. It should be noted that our study focused on experimental designs in relation to intrarater reliability. It is also important to understand the effect of pooling estimates of the same specimen by different raters (i.e., interrater reliability effects) in future studies.
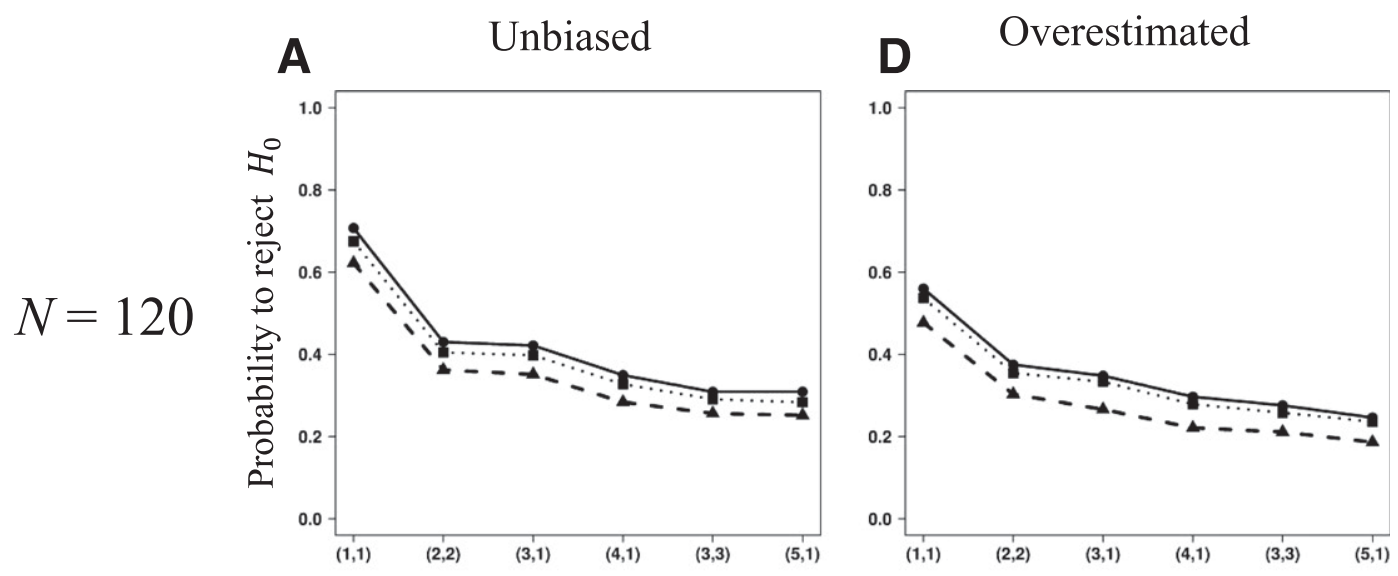

B

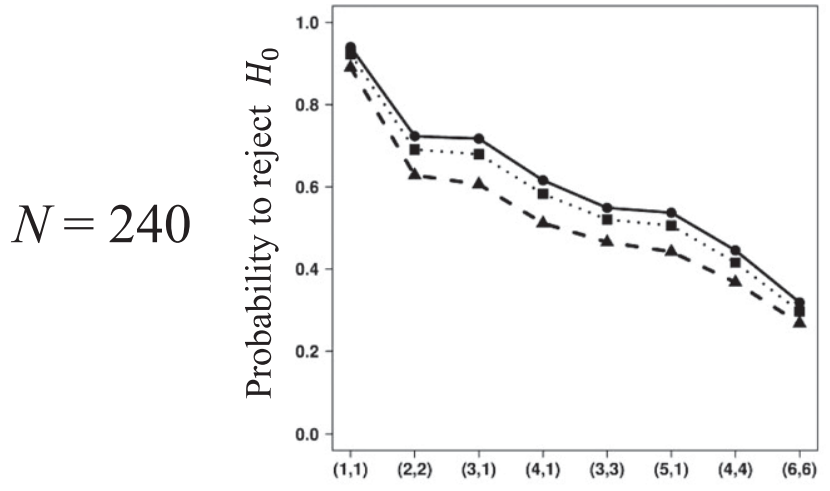

C
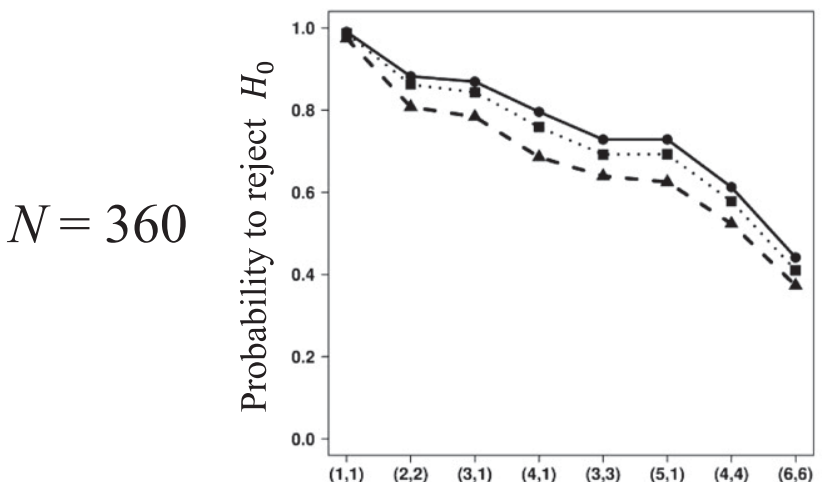

E

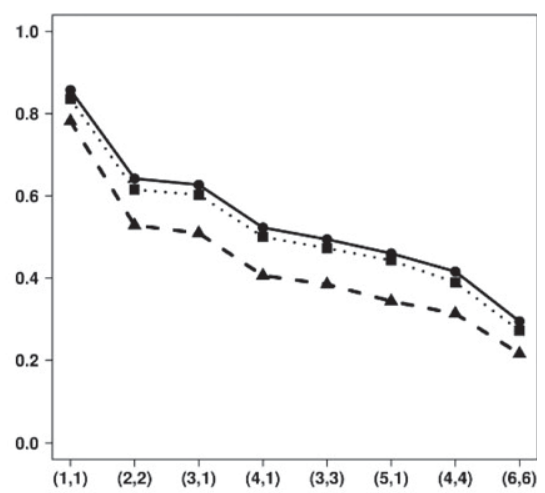

$\mathbf{F}$

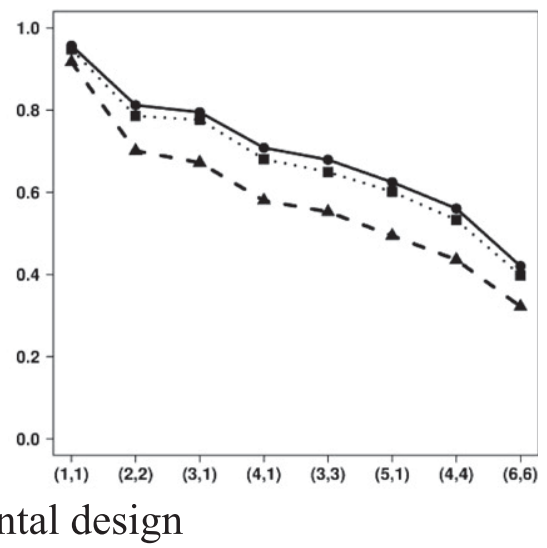

Experimental design

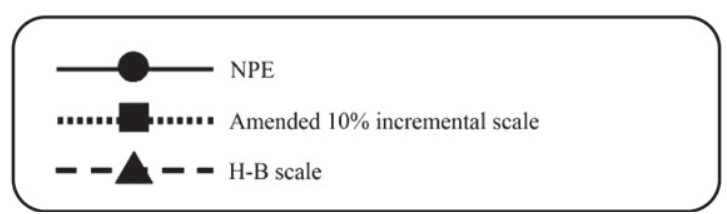

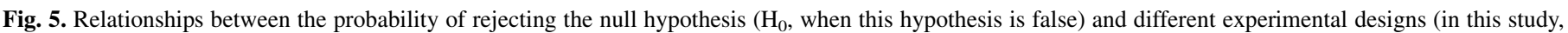

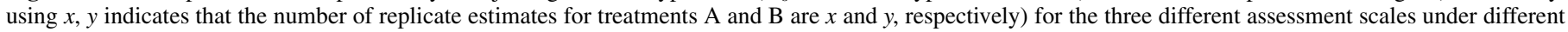

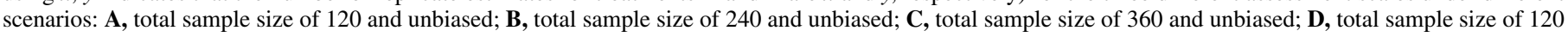

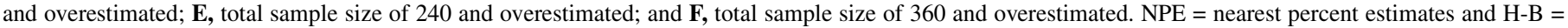
Horsfall-Barratt. Difference between the population means $\left(\mu_{\Delta}\right)$ is assumed to be $5 \%, \varphi=10 \%$, and $\mu_{\mathrm{A}}=20 \%$, with significance at $P=0.05$. 
Obtaining two replicate estimates of each specimen by the same rater might not be practical in all situations. However, in situations where specimens are collected from the field for later assessment or tagged in situ, or if assessments are performed on known individuals in a greenhouse or growth-chamber experiment, it should be practical to apply the proposed approach. By assessing each specimen twice in an arbitrary or random order, the rater will be able to provide two independent estimates of the same sample while maintaining the
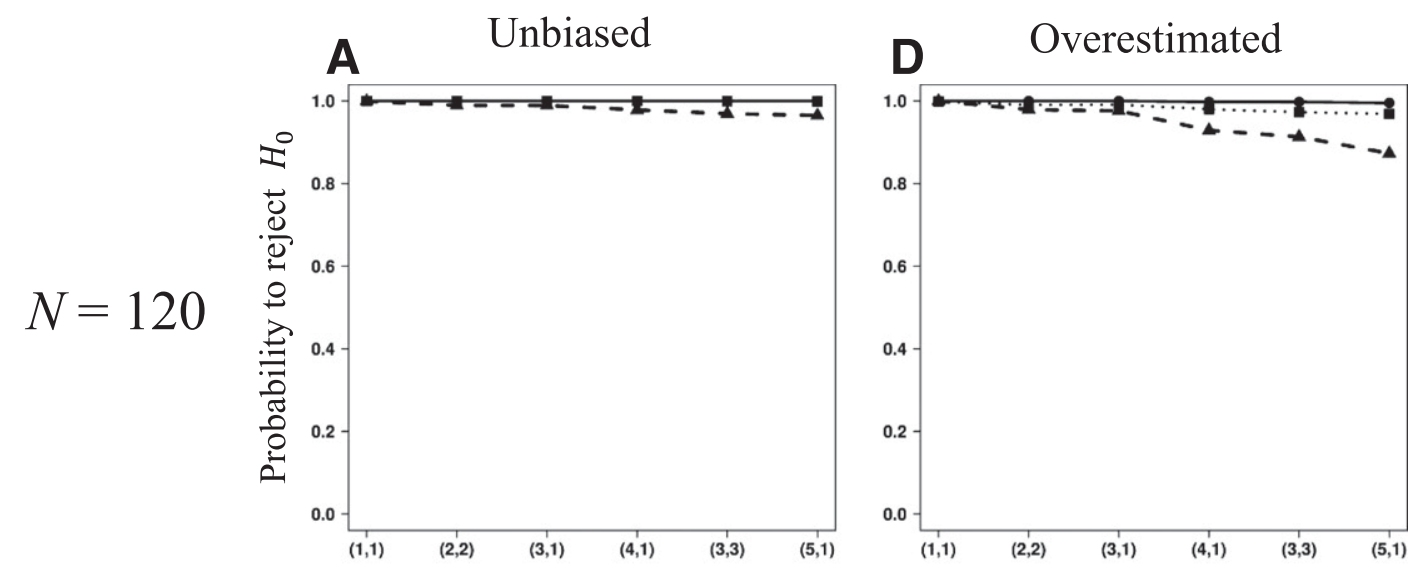

B

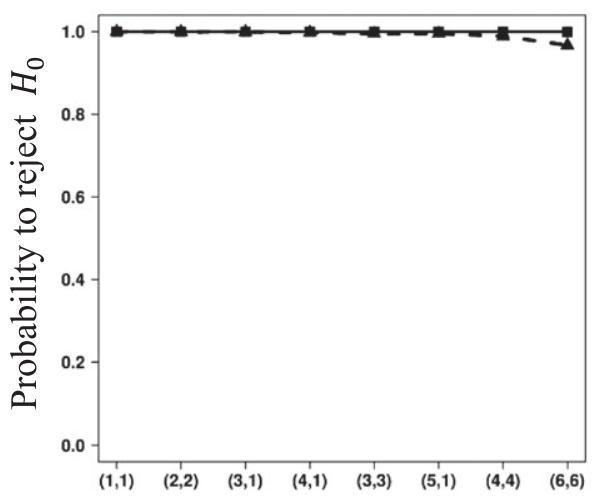

C

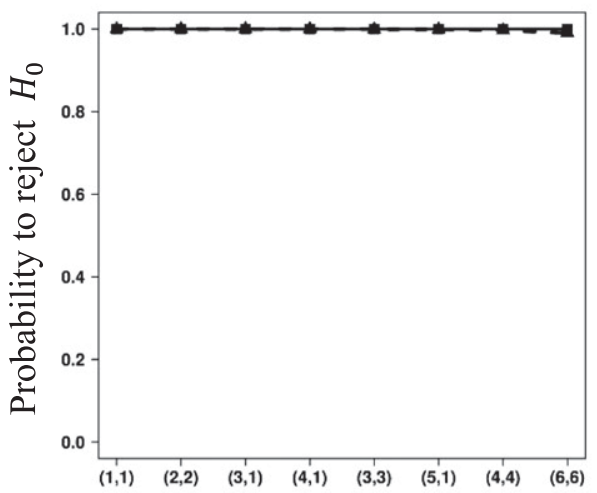

E

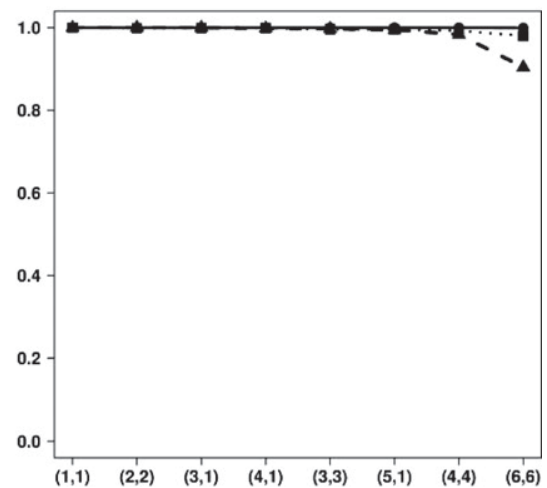

$\mathbf{F}$

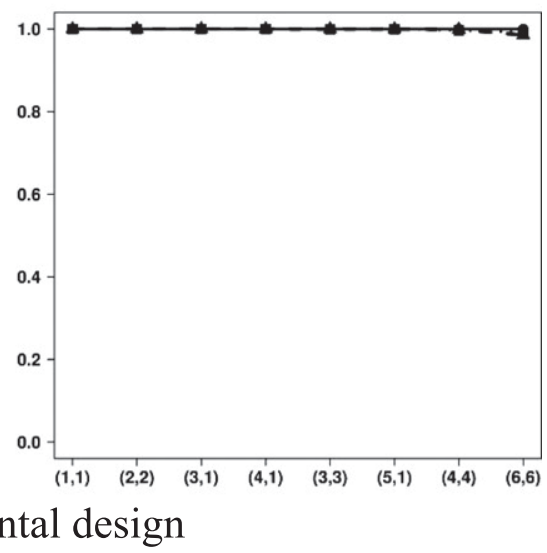

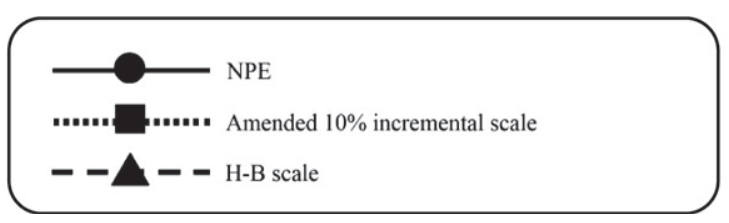

Fig. 6. Relationships between the probability of rejecting the null hypothesis $\left(\mathrm{H}_{0}\right.$, when this hypothesis is false) and different experimental designs (in this study, using $x, y$ indicates that the number of replicate estimates for treatments $\mathrm{A}$ and $\mathrm{B}$ are $x$ and $y$, respectively) for the three different assessment scales under different scenarios: A, total sample size of 120 and unbiased; B, total sample size of 240 and unbiased; C, total sample size of 360 and unbiased; D, total sample size of 120 and overestimated; $\mathbf{E}$, total sample size of 240 and overestimated; and $\mathbf{F}$, total sample size of 360 and overestimated. NPE = nearest percent estimates and H-B = Horsfall-Barratt. Difference between the population means $\left(\mu_{\Delta}\right)$ is assumed to be $10 \%, \varphi=5 \%$, and $\mu_{\mathrm{A}}=20 \%$, with significance at $P=0.05$. 
same statistical power overall (a process of random or arbitrary selection of the specimen with a temporal separation to prevent prior estimation bias should allow for two independent estimates).

However, characteristics of a rater will affect recommended sample size. For example, if a rater overestimates and uses the $10 \%$ incremental scale, that rater will have to increase the total sample size for the treatments being compared to approximately 240 in order to obtain a high power of discrimination between treatments. In this case, when the number of specimens collected equals 60 and two replicate estimates are taken per specimen, the power
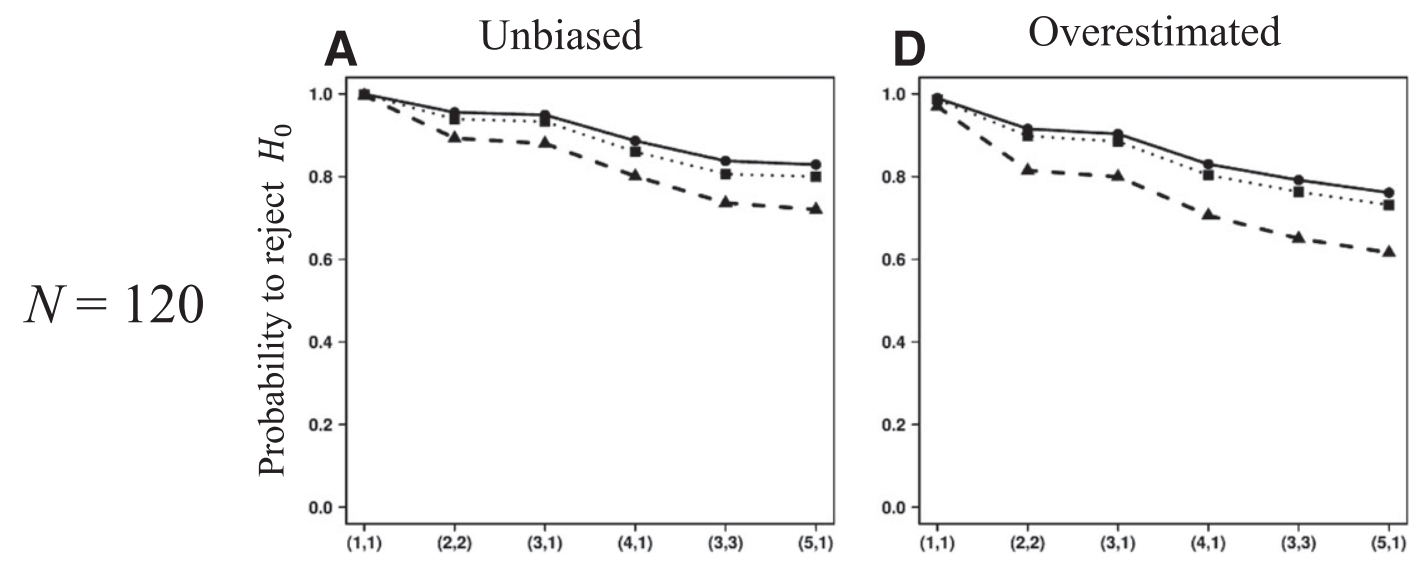

B

E
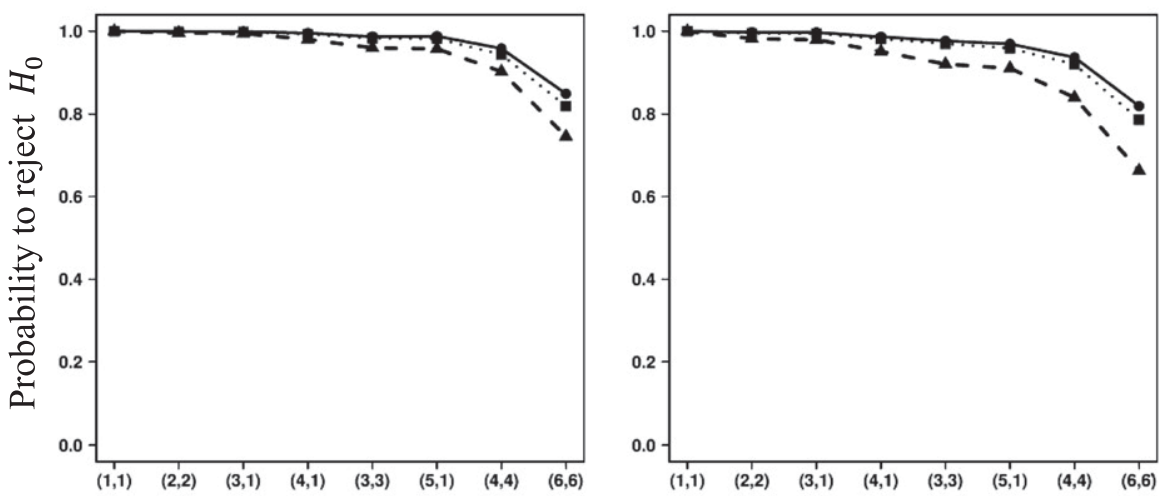

C

$\mathbf{F}$

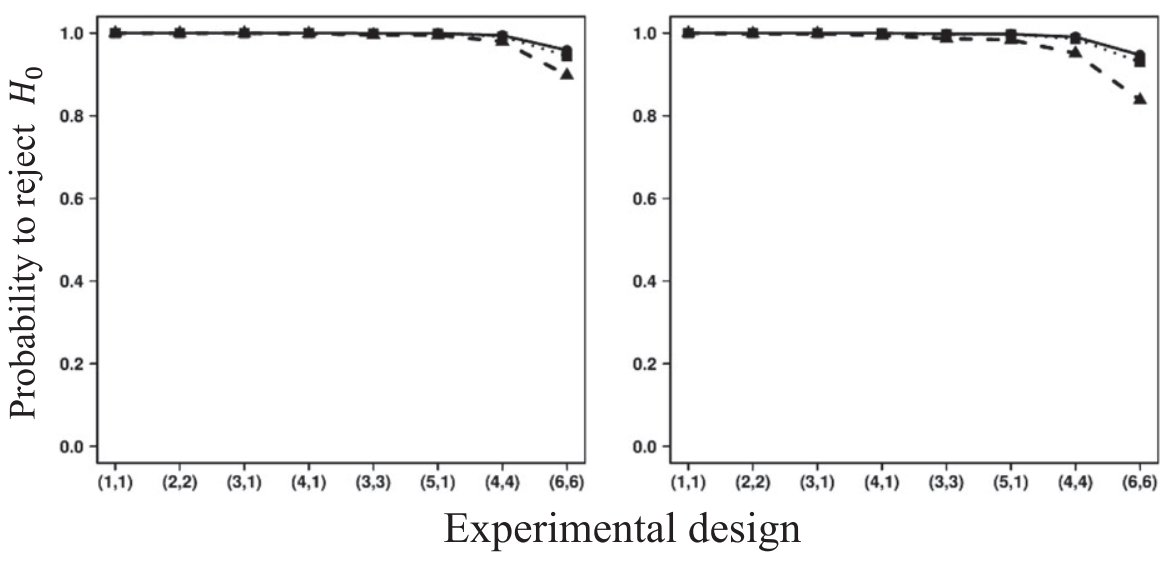

$N=360$

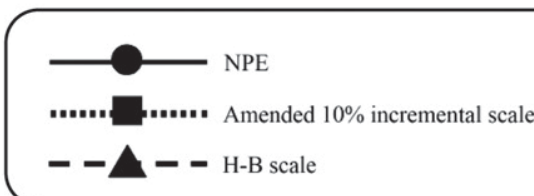

Fig. 7. Relationships between the probability of rejecting the null hypothesis $\left(\mathrm{H}_{0}\right.$, when this hypothesis is false) and different experimental designs (in this study, using $x, y$ indicates that the number of replicate estimates for treatments A and $\mathrm{B}$ are $x$ and $y$, respectively) for the three different assessment scales under different scenarios: A, total sample size of 120 and unbiased; B, total sample size of 240 and unbiased; C, total sample size of 360 and unbiased; D, total sample size of 120 and overestimated; $\mathbf{E}$, total sample size of 240 and overestimated; and $\mathbf{F}$, total sample size of 360 and overestimated. NPE $=$ nearest percent estimates and H-B = Horsfall-Barratt. Difference between the population means $\left(\mu_{\Delta}\right)$ is assumed to be $10 \%, \varphi=10 \%$, and $\mu_{\mathrm{A}}=20 \%$, with significance at $P=0.05$. 
approaches $100 \%$. These rater estimates of each specimen severity might not only affect the resulting mean value but also affect the variance (and $\varphi$ ) of that mean value. The hypothesis test relies not only on accurate mean values but also on the sample variance being a true representation of the population variance.
Although the greatest power observed for the test was for the balanced design $(1,1)$, this design uses only a single replicate for each specimen from each treatment (and, thus, does not use replicate estimates of the same specimen to stabilize the mean and variance in the analysis). Greater numbers of sampling units will
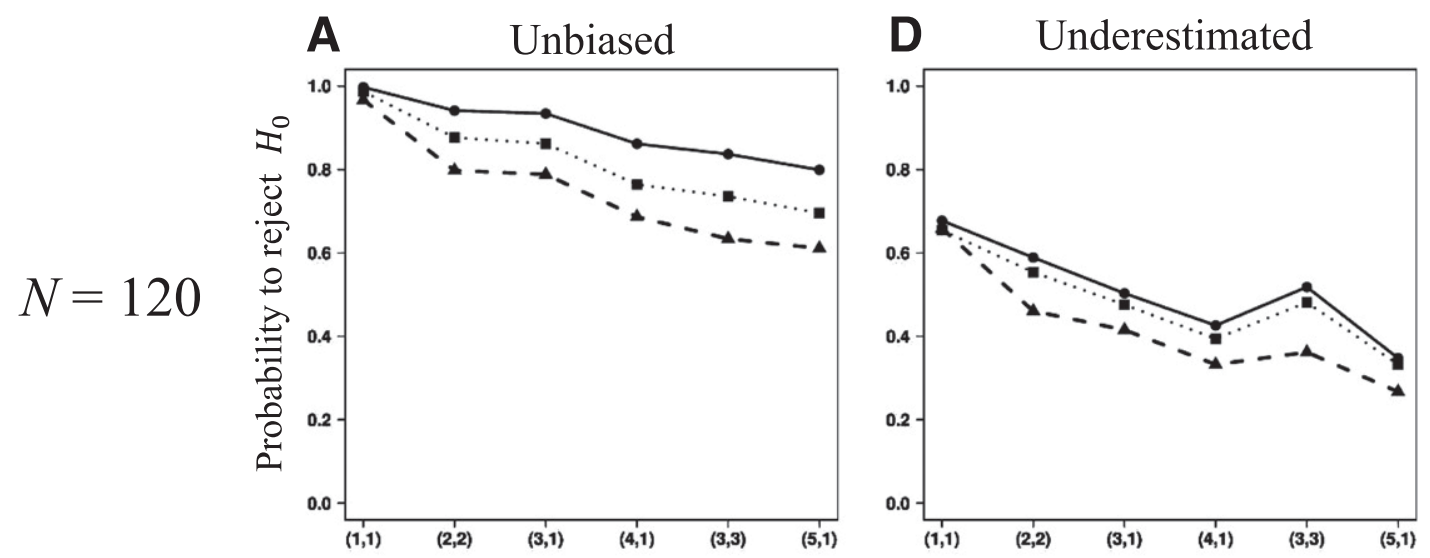

B

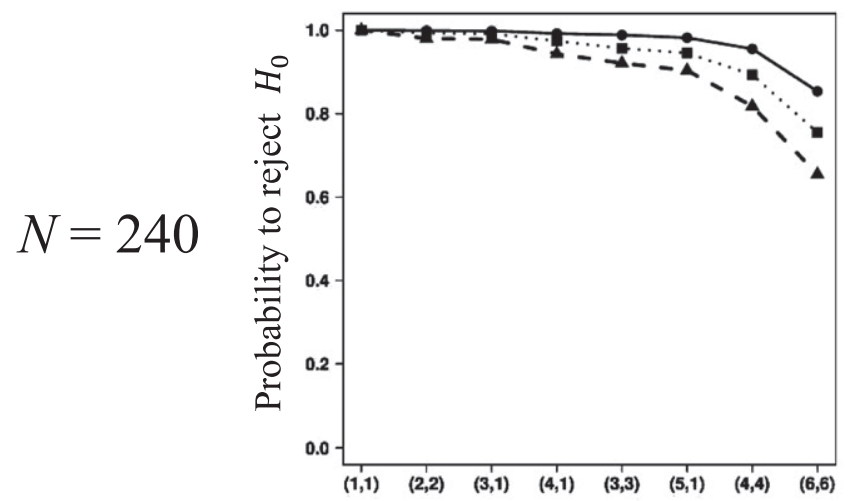

C
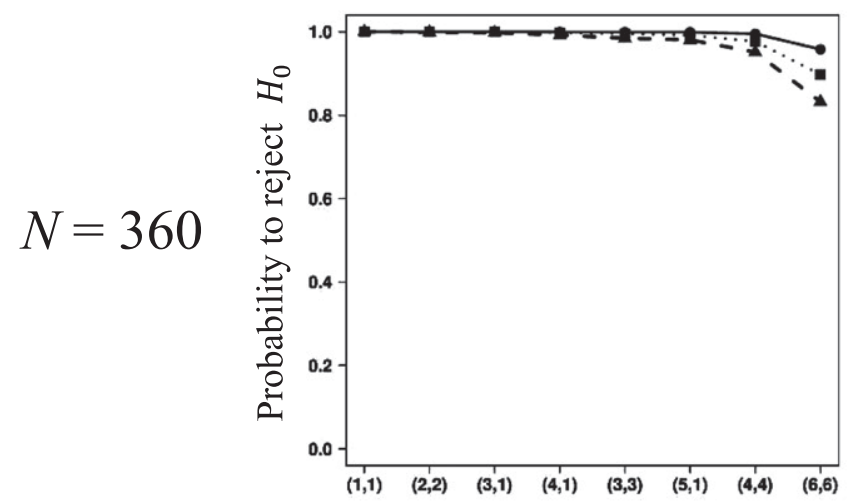

E

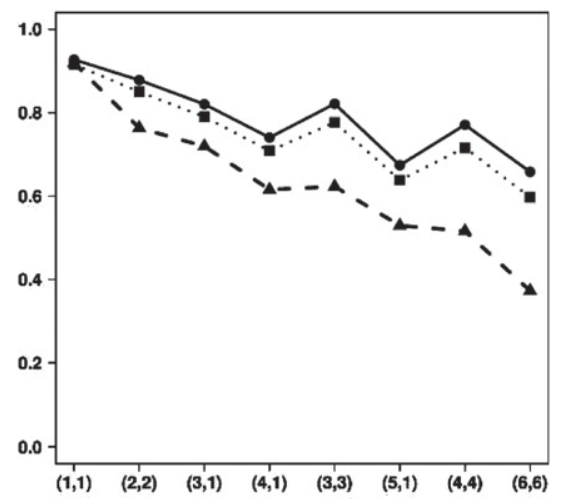

$\mathbf{F}$

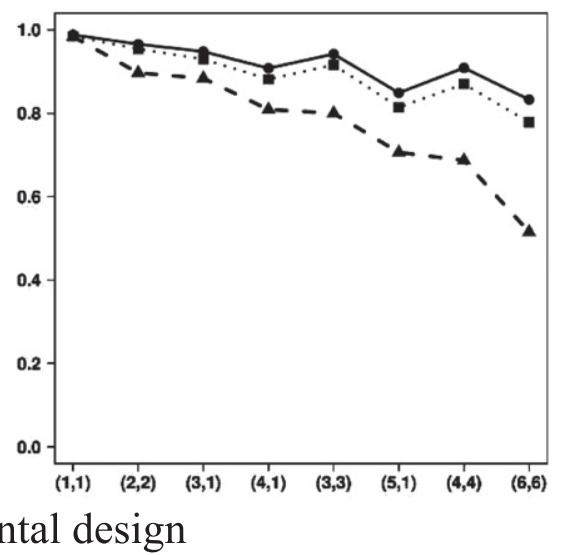

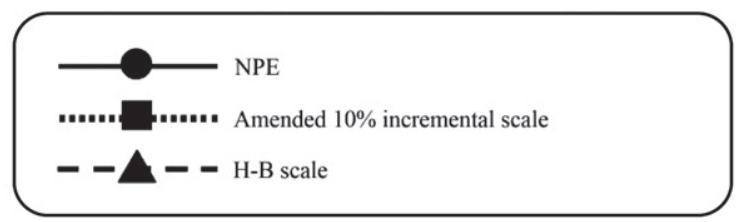

Fig. 8. Relationships between the probability of rejecting the null hypothesis $\left(\mathrm{H}_{0}\right.$, when this hypothesis is false) and different experimental designs (in this study, using $x, y$ indicates that the number of replicate estimates for treatments $\mathrm{A}$ and $\mathrm{B}$ are $x$ and $y$, respectively) for the three different assessment scales under different scenarios: A, total sample size of 120 and unbiased; B, total sample size of 240 and unbiased; C, total sample size of 360 and unbiased; $\mathbf{D}$, total sample size of 120 and underestimated; $\mathbf{E}$, total sample size of 240 and underestimated; and $\mathbf{F}$, total sample size of 360 and underestimated. NPE $=$ nearest percent estimates and H-B = Horsfall-Barratt. Difference between the population means $\left(\mu_{\Delta}\right)$ is assumed to be $5 \%, \varphi=5 \%$, and $\mu_{\mathrm{A}}=75 \%$, with significance at $P=0.05$. 
require increases in both time (labor) and money (Nutter and Esker 2006; Nutter and Gaunt 1996). Therefore, the results and rationale suggest that two replicate estimates per specimen (the number of specimens collected should be at least 30) are more efficient in experimental designs where plant disease assessments are made. It is also consistent with the test-retest procedure in previous studies of plant disease assessment (Bock et al. 2008a,b; Duarte et al. 2013; Nita et al. 2003; Shokes et al. 1987). With limited resources, cost is an important consideration in plant pathological studies and was a recently stated reason for developing a novel population-based
A

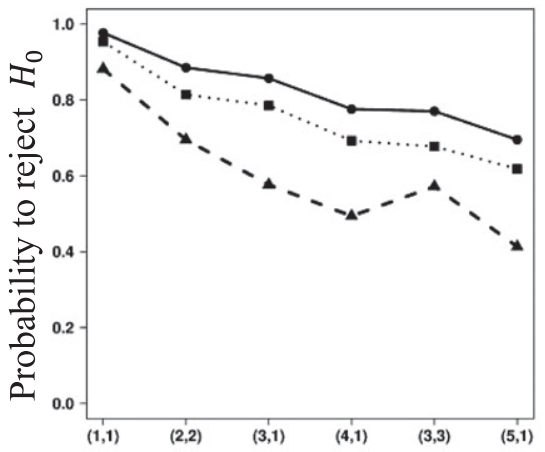

B

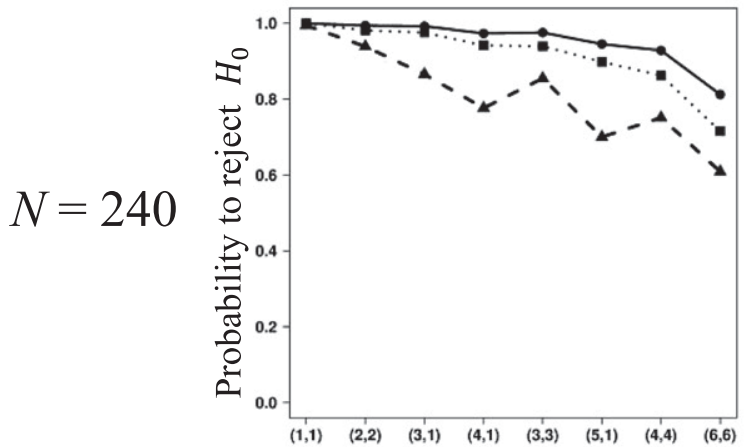

C

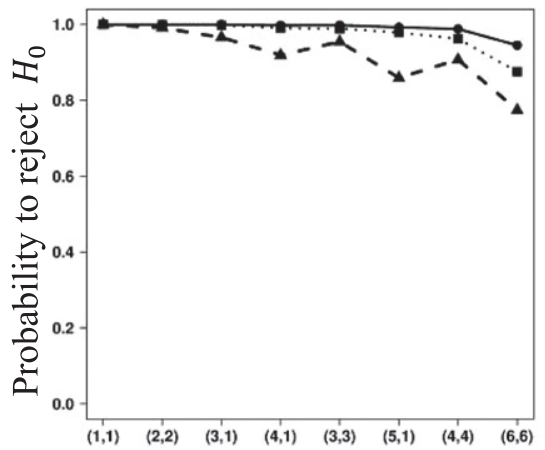

D Overestimated

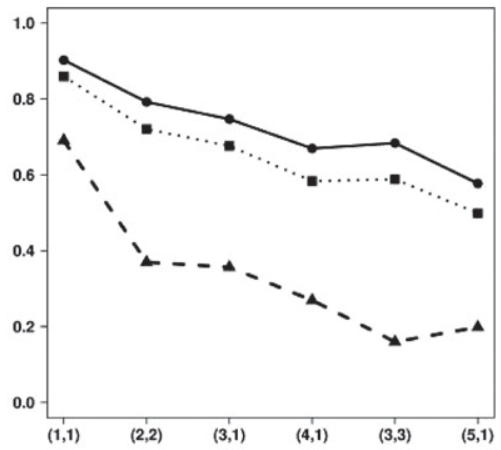

E

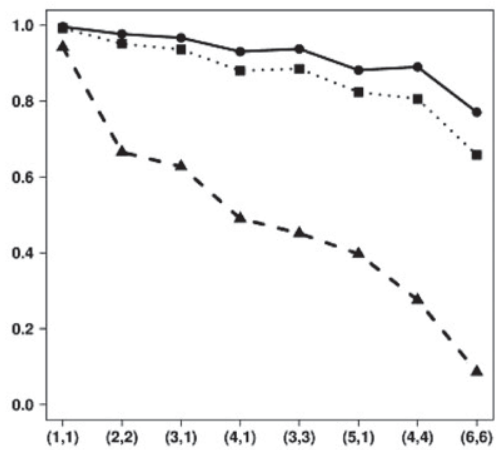

$\mathbf{F}$

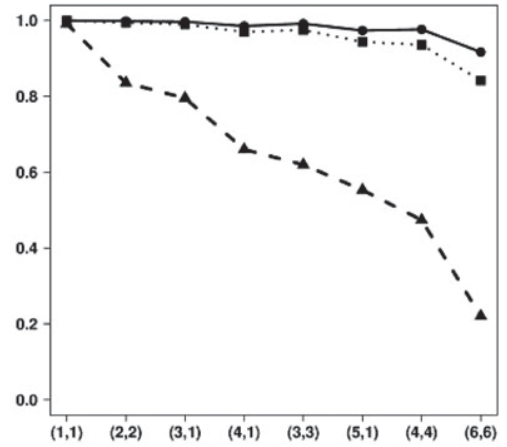

\section{G Underestimated}

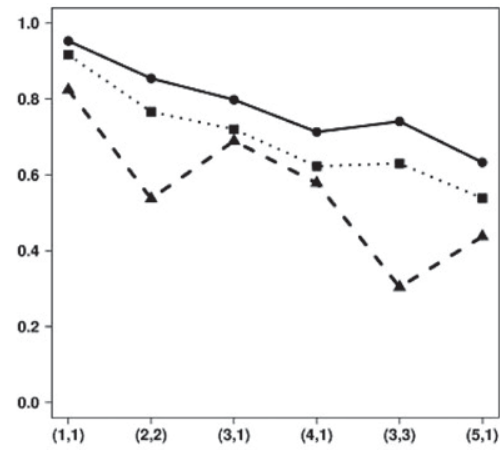

H

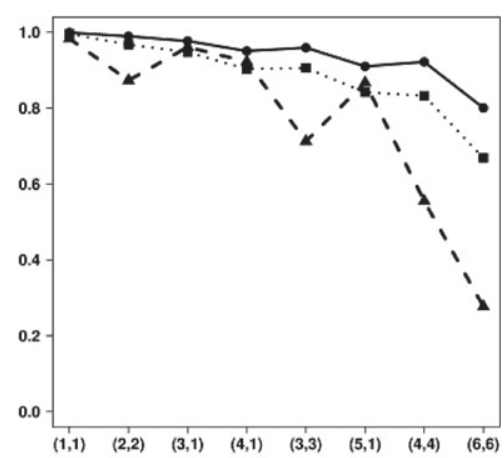

I

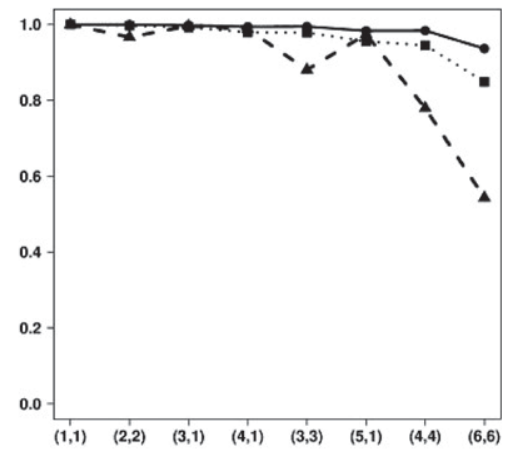

\section{Experimental design}

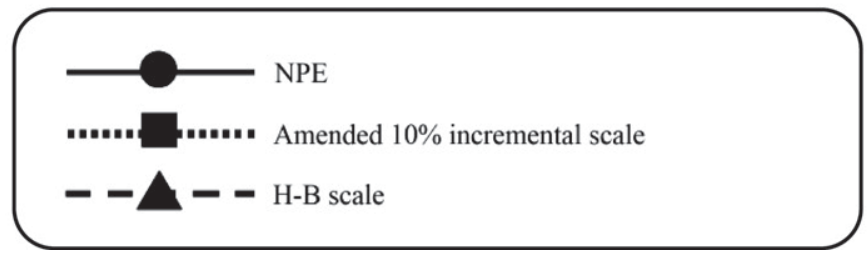

Fig. 9. Relationships between the probability of rejecting the null hypothesis $\left(\mathrm{H}_{0}\right.$, when this hypothesis is false) and different experimental designs (in this study, using $x, y$ indicates that the number of replicate estimates for treatments A and $\mathrm{B}$ are $x$ and $y$, respectively) for the three different assessment scales under different scenarios: A, total sample size of 120 and unbiased; B, total sample size of 240 and unbiased; C, total sample size of 360 and unbiased; D, total sample size of 120 and overestimated; $\mathbf{E}$, total sample size of 240 and overestimated; F, total sample size of 360 and overestimated; $\mathbf{G}$, total sample size of 120 and underestimated; $\mathbf{H}$, total sample size of 240 and underestimated; and $\mathbf{I}$, total sample size of 360 and underestimated. NPE = nearest percent estimates and H-B = Horsfall-Barratt. Difference between the population means $\left(\mu_{\Delta}\right)$ is assumed to be $5 \%, \varphi=5 \%$, and $\mu_{\mathrm{A}}=50 \%$, with significance at $P=0.05$. 
method to estimate disease severity (Bousset et al. 2016). Therefore, the need to conserve resources through optimizing experiment design is compelling.

In this article, the number of specimens was assumed to be the total infected leaves observed or collected from a single treatment plot. In most cases, an experiment will consist of several blocks (with one or more plots per treatment in each block) (e.g., a randomized complete block design) (Bock et al. 2015; Todd and Kommedahl 1994). In general, the same number of specimens is collected from each block. For example, there were two treatments and four blocks with the upper three leaves on five wheat stems per block being assessed in the experiment on which these leaf samples were based (Bock et al. 2015; El Jarroudi et al. 2015). However, in other cases, the number of specimens collected from each block may not be even. How many specimens will be collected from each block? We need to test whether the distribution of the diseased status has the same variability per block. Bartlett's test and Levene's test are recommended to gauge the variability (Shoukri and Pause 1999). It would seem reasonable to take larger samples from the more variable blocks and smaller samples from the less variable blocks. The sample size arising from this kind of allocation is called optimum allocation (Freund and Perles 2006). This, too, is an area for future studies.

The interacting effects of assessment methods and other factors on hypothesis testing. Although the amended $10 \%$ scale has slightly less power compared with NPEs, it has greater congruence with that of NPEs compared with the H-B and H-B-related scales. A further valuable observation is that the H-B scale had a lower power of the test compared with all other assessment methods used at all replicate numbers tested (whether based on balanced or unbalanced data sets). In using the $\mathrm{H}-\mathrm{B}$ scale, results based on a balanced experimental design are superior to an unbalanced experimental design in most cases. However, there are some exceptions: the balanced design was not superior using the H-B scale when severity was overestimated or underestimated at a $\mu_{\mathrm{A}}$ of $50 \%$. The exception does not exist in unbiased data. It can be ascribed to the wider interval ( 25 to 50 or 50 to $75 \%$ ) of the H-B scale over these midrange severities. For example, for the experimental design $(4,1)$, there is only one replicate for treatment $\mathrm{B}$. When underestimated, the rater estimate for treatment $\mathrm{B}$ is $37.5 \%$ (the midpoint of 25 to $50 \%$ ). Thus, it is easier to detect a difference between the means. At a mean severity of 5\%, powers of the $\mathrm{H}-\mathrm{B}$ scale are similar to those found for raters using the other assessment methods. Again, the reason is that the $\mathrm{H}-\mathrm{B}$ scale has narrower intervals at low severities $\left(0^{+}\right.$to $3,3^{+}$to 6 , and so on) (Bock et al. 2010a; Chiang et al. 2014; Nutter and Esker 2006).

Effects of biased estimates and other factors on hypothesis testing. For biased estimates, a noticeable difference in the power of the hypothesis test was observed between different assessment methods and between different experimental designs. When the $\varphi$ was increased from 5 to $10 \%$, the power of the hypothesis test declined for all experimental designs, unbiased and biased raters, and assessment methods. However, when the $\mu_{\Delta}$ was increased to $10 \%$, the power of the test approached 1 and, not unexpectedly, it was easier to detect the differences between the means. These results suggest that it is usually better to avoid using an unbalanced experiment design, particularly when combined with the H-B scale method of assessment (or other category scales with similarly structured uneven intervals); the power of the test is reduced yet further if the rater has a tendency to biased estimates.

Rationale for simulation studies. Inevitably, the simulation approach we used is an approximation to the actual factors governing specific situations of pathosystems and disease severities, raters, sampling protocols (which were not an objective of this study), and experimental designs (as well as other factors) in relation to hypothesis testing. For each specimen, estimates of severity will most often be different from the actual severity, which results in absolute error. Absolute error accumulated over the sample of specimens can affect the sample mean and variance when compared with the actual population mean and variance. The simulation approach used here aimed to mimic the process of disease severity estimation. For example, if an actual severity value ( $\left.Y_{\text {actual }}\right)$ of $45 \%$ was selected, a simulated estimate of $\mu_{\mathrm{A}}=40 \%$ might be made for treatment $A$. In this case, because the actual mean of the generalized rater estimation distribution (a lognormal distribution $)=$ $45 \%$, various simulated specimen values (e.g., 47, 41, 37\%, and so on) can be drawn from that lognormal distribution. Thus, the difference between the above simulated value (e.g., 47, 41, 37\%, and so on) and $Y_{\text {actual }}(45 \%)$ is the error rate in assessment of the mean (repeated assessment of specimens) and that mimics rater estimate effects on the variance and $\varphi$ of the sample. Moreover, the difference between $\mu_{\mathrm{A}}$ $(40 \%)$ and $Y_{\text {actual }}(45 \%)$ is the variation in infection among individuals in a field plot. Furthermore, an actual severity $\left(Y_{\text {actual }}\right)$ of 30 or even $20 \%$ could also be selected during the simulation process, because the population (A or B) is normally distributed to mimic variation in infection among individuals in a field plot population. Thus, both the variation in infection among individuals in a field plot and the error rate in assessment were considered in the simulation process.

A perspective from the medical literature. In the medical literature, similar studies have investigated sample size requirements for the purpose of designing experiments to explore reliability (Eliasziw and Donner 1987; Flynn et al. 2002; Giraudeau and Mary 2001; Shoukri 2004). The purpose of these studies is to determine the optimum number of specimens and replicate estimates per specimen so that the variance of the estimator for the intraclass correlation coefficient is minimized; as in plant pathology studies, the total number of observations is constrained by resource limitations. The conclusion from the studies in the medical literature is that two or three replicate estimates per specimen is recommended to provide a reliability of at least $60 \%$ to ensure a useful method of measurement (Shoukri 2004). In addition, in medical studies, it may be less costly to increase the number of replicate estimates per specimen than to increase the number of specimens. Thus, a larger number of replicate estimates could be expected. With the increase of the number of replicate estimates, the number of specimens decreases; this will lead to a loss in precision of the estimated reliability. Therefore, two or three replicate estimates per specimen are recommended in medical studies. These results are consistent with our own observations from the current study.

Conclusion. The results of our study suggest that the number of replicate estimates of individual samples and the choice of experimental design are crucial to maximizing the probability of correctly differentiating treatments where disease severity estimates will be used for hypothesis testing. That is, with unbiased estimates using NPEs, the recommended number of replicate estimates taken per specimen is two (from a sample of at least 30 specimens), because this conserves resources. It is usually better to avoid using an unbalanced experiment design, particularly when combined with the H-B scale method of assessment (or other category scales with similarly structured uneven intervals); the power of the test is reduced yet further if the rater has a tendency to biased estimates. These recommendations are basically general and, thus, applicable across most or many plant disease assessment situations.

In conclusion, we hope that, by demonstrating the effects of different experimental designs, methods of assessment, and effects of rater bias, we bring useful insights that will allow plant pathologists to better select options in the experimental process that minimize the risk of type II errors. Furthermore, based on this understanding, development and use of assessment aids can be promoted to help minimize this source of error.

\section{ACKNOWLEDGMENTS}

We thank F. van den Bosch, S. Parnell, and T. Gottwald for many discussions on aspects of plant disease assessment that provided a basis for developing this work. K.-S. Chiang gratefully acknowledges financial support from the Ministry of Science and Technology of Taiwan, R.O.C. (MOST 104-2313-B-005-027 and MOST 105-2313-B-005-013). C. Bock acknowledges support for the research through the United States Department of Agriculture (USDA) Agricultural Research Service project 
number 6042-21220-012-00. M. El Jarroudi and P. Delfosse thank the Administration des Services Techniques de l'Agriculture, Luxembourg for funding the MACRY project. This article reports the results of research only. Mention of a trademark or proprietary product is solely for the purpose of providing specific information and does not constitute a guarantee or warranty of the product by the USDA and does not imply its approval to the exclusion of other products that may also be suitable.

\section{LITERATURE CITED}

Bock, C. H., El Jarroudi, M., Kouadio, A. L., Mackels, C., Chiang, K. S., and Delfosse, P. 2015. Disease severity estimates-Effects of rater accuracy and assessment methods for comparing treatments. Plant Dis. 99:1104-1112.

Bock, C. H., Gottwald, T. R., Parker, P. E., Ferrandino, F., Welham, S., van den Bosch, F., and Parnell, S. 2010a. Some consequences of using the Horsfall-Barratt scale for hypothesis testing. Phytopathology 100:1030-1041.

Bock, C. H., Parker, P. E., Cook, A. Z., and Gottwald, T. R. 2008a. Visual rating and the use of image analysis for assessing different symptoms of citrus canker on grapefruit leaves. Plant Dis. 92:530-541.

Bock, C. H., Parker, P. E., Cook, A. Z., and Gottwald, T. R. 2008b. Characteristics of the perception of different severity measures of citrus canker and the relationships between the various symptom types. Plant Dis. 92:927-939.

Bock, C. H., Poole, G. H., Parker, P. E., and Gottwald, T. R. 2010b. Plant disease severity estimated visually, by digital photography and image analysis, and by hyperspectral imaging. Crit. Rev. Plant Sci. 29:59-107.

Bousset, L., Jumel, S., Picault, H., Domin, C., Lebreton, L., Ribule, A., and Delourme, R. 2016. An easy, rapid and accurate method to quantify plant disease severity: Application to Phoma stem canker leaf spots. Eur. J. Plant Pathol. 145:697-709.

Campbell, L. C., and Madden, L. V. 1990. Introduction to Plant Disease Epidemiology. John Wiley \& Sons, New York.

Chiang, K. S., Bock, C. H., El Jarroudi, M., Delfosse, P., Lee, I. H., and Liu, H. I. 2016. Effects of rater bias and assessment method on disease severity estimation with regard to hypothesis testing. Plant Pathol. 65:523-535.

Chiang, K. S., Liu, S. H., Bock, C. H., and Gottwald, T. R. 2014. What interval characteristics make a good disease assessment category scale? Phytopathology 104:575-585.

Duarte, H. S. S., Zambolin, L., Capucho, A. S., Nogueira, A. F., Rosado, A. W. C., Cardoso, C. R., Paul, P. A., and Mizubuti, E. S. G. 2013. Development and validation of a set of standard area diagrams to estimate severity of potato early blight. Eur. J. Plant Pathol. 137:249-257.

Eliasziw, M., and Donner, A. 1987. A cost-function approach to the design of reliability studies. Stat. Med. 6:647-655.

El Jarroudi, M., Kouadio, A. L., Mackels, C., Tychon, B., Delfosse, P., and Bock, C. H. 2015. A comparison between visual estimates and image analysis measurements to determine Septoria leaf blotch severity in winter wheat. Plant Pathol. 64:355-364.

Everitt, B. S. 1998. Dictionary of Statistics. Cambridge University Press, Cambridge.

Flynn, N. T., Whitley, E., and Peters, T. 2002. Recruitment strategy in a cluster randomized trial-Cost implications. Stat. Med. 21:397-405.

Forbes, G. A., and Korva, J. T. 1994. The effect of using a Horsfall-Barratt scale on precision and accuracy of visual estimation of potato late blight severity in the field. Plant Pathol. 43:675-682.
Freund, J. E., and Perles, B. M. 2006. Modern Elementary Statistics, 12th ed. Pearson Prentice Hall, Upper Saddle River, NY.

Giraudeau, B., and Mary, J. Y. 2001. Planning a reproducibility study: How many subjects and how many replicates per subject for an expected width of the 95 percent confidence interval of the intraclass correlation coefficient. Stat. Med. 20:3205-3214.

Godoy, C. V., Koga, L. J., and Canteri, M. G. 2006. Diagrammatic scale for assessment of soybean rust severity. Fitopatol. Bras. 31:63-68.

Horsfall, J. G., and Barratt, R. W. 1945. An improved grading system for measuring plant disease. (Abstr.) Phytopathology 35:655.

Madden, L. V., Hughes, G., and van den Bosch, F. 2007. The Study of Plant Disease Epidemics. American Phytopathological Society, St. Paul, MN.

Martin, D. P., and Rybicki, E. P. 1998. Microcomputer-based quantification of maize streak virus symptoms in Zea mays. Phytopathology 88:422-427.

Montgomery, D. C. 2005. Design and Analysis of Experiments, 6th ed. Wiley, New York

Nita, M., Ellis, M. A., and Madden, L. V. 2003. Reliability and accuracy of visual estimation of Phomopsis leaf blight of strawberry. Phytopathology 93:995-1005.

Nutter, F. W., Jr., and Esker, P. D. 2006. The role of psychophysics in phytopathology: The Weber-Fechner law revisited. Eur. J. Plant Pathol. 114: 199-213.

Nutter, F. W., Jr., and Gaunt, R. E. 1996. Recent developments in methods for assessing disease losses in forage/pasture crops. Pages 93-118 in: Pasture and Forage Crop Pathology. S. Chakraborty, ed. ASA, CSSA, and SSSA, Madison, WI.

Nutter, F. W., Jr., Gleason, M. L., Jenco, J. H., and Christians, N. C. 1993. Assessing the accuracy, intra-rater repeatability, and inter-rater reliability of disease assessment system. Phytopathology 83:806-812.

Nutter, F. W., Jr., and Schultz, P. M. 1995. Improving the accuracy and precision of disease assessment: Selection of methods and use of computeraided training programs. Can. J. Plant Pathol. 17:174-184.

Nutter, F. W., Jr., Teng, P. S., and Shokes, F. M. 1991. Disease assessment terms and concepts. Plant Dis. 75:1187-1188.

Parker, S. R., Shaw, M. W., and Royle, D. J. 1995. The reliability of visual estimates of disease severity on cereal leaves. Plant Pathol. 44:856-864.

Sherwood, R. T., Berg, C. C., Hoover, M. R., and Zeiders, K. E. 1983. Illusions in visual assessment of Stagonospora leaf spot of orchardgrass. Phytopathology 73:173-177.

Shokes, F. M., Berger, R. D., Smith, D. H., and Rasp, J. M. 1987. Reliability of disease assessment procedures: A case study with late spot of peanut. Oleagineux 42:245-251.

Shoukri, M. M. 2004. Measures of Interobserver Agreement, 2nd ed. Chapman \& Hall/CRC Press, Boca Raton, FL.

Shoukri, M. M., Asyali, M. H., and Walter, S. D. 2003. Issues of cost and efficiency in the design of reliability studies. Biometrics 59:1107-1112.

Shoukri, M. M., and Pause, C. A. 1999. Statistical Methods for Health Science, 2nd ed. CRC Press, Boca Raton, FL.

Steel, R. G. D., Torrie, J. H., and Dickey, D. A. 1997. Principles and Procedures of Statistics: A Biometrical Approach, 3rd ed. McGraw-Hill, New York.

Todd, L. R., and Kommedahl, T. 1994. Image analysis and visual estimates for evaluating disease reactions of corn to Fusarium stalk rot. Plant Dis. 78: 876-878. 